NBER WORKING PAPER SERIES

\title{
CHANGES IN ASSORTATIVE MATCHING: THEORY AND EVIDENCE FOR THE US
}

Pierre-André Chiappori

Monica Costa Dias

Costas Meghir

Working Paper 26932

http://www.nber.org/papers/w26932

\author{
NATIONAL BUREAU OF ECONOMIC RESEARCH \\ 1050 Massachusetts Avenue \\ Cambridge, MA 02138 \\ April 2020, Revised April 2020
}

Paper presented at seminars and conferences at Stony Brook, University of Pennsylvania, Oxford University, University of Essex and University of Bristol; we thank the participants, Bernard Salanié and Dan Anderberg for their comments. Financial support from the ESRC Centre for the Microeconomic Analysis of Public Policy (CPP) at IFS and from the ESRC awards ES/ K00624X/1, ES/N015304/1 is gratefully acknowledged. Costas Meghir also thanks the Cowles Foundation and the ISPS at Yale for financial support. All errors are our own. The views expressed herein are those of the authors and do not necessarily reflect the views of the National Bureau of Economic Research.

NBER working papers are circulated for discussion and comment purposes. They have not been peer-reviewed or been subject to the review by the NBER Board of Directors that accompanies official NBER publications.

(C) 2020 by Pierre-André Chiappori, Monica Costa Dias, and Costas Meghir. All rights reserved. Short sections of text, not to exceed two paragraphs, may be quoted without explicit permission provided that full credit, including $(\odot$ notice, is given to the source. 
Changes in Assortative Matching: Theory and Evidence for the US

Pierre-André Chiappori, Monica Costa Dias, and Costas Meghir

NBER Working Paper No. 26932

April 2020, Revised April 2020

JEL No. J01,J11,J12

\section{$\underline{\text { ABSTRACT }}$}

The extent to which like-with like marry is particularly important for inequality as well as for the outcomes of children that result from the union. In this paper we discuss approaches to the measurement of changes in assortative mating. We derive two key conditions that a well-defined measure should satisfy. We argue that changes in assortativeness should be interpreted through a structural model of the marriage market; in particular, a crucial issue is how they relate to variations in the economic surplus generated by marriage. We propose a very general criterion of increase in assortativeness, and show that almost all indices used in the literature are implied by our criterion with one notable exception, that moreover violates one of our conditions. Finally, we use our approach to evaluate the evolution of assortative matching in the US over the last decades, and conclude that assortative matching has increased, particularly at the top of the education distribution.

Pierre-André Chiappori

Department of Economics

Columbia University

1009A International Affairs Building

420 West 118th St.

New York, NY 10027

pc2167@columbia.edu

Monica Costa Dias

Institute For Fiscal Studies

7, Ridgmount Street

London WC1E 7AE, UK

monica_d@ifs.org.uk
Costas Meghir

Department of Economics

Yale University

37 Hillhouse Avenue

New Haven, CT 06511

and IZA

and also NBER

c.meghir@yale.edu 


\section{Introduction}

The study of sorting in the marriage market has recently attracted renewed attention because of its potential role in determining changing inequality and because college attainment has changed substantially, with implications for the marriage market. More broadly, sorting has consequences for family formation and intergenerational transmission of human capital so understanding how it is changing can be central to the study of inequality and intergenerational mobility. For example, to the extent that male and female human capital are complementary in raising children and determining their abilities, an increase in sorting will likely lead to increased inequality in human capital in future generations. On the other hand, it is precisely these complementarities and the well documented increase in the returns to human capital that may underlie the increased sorting.

Whatever the reason for the potential changes in sorting, and indeed whatever its implications, it is important to be able to measure it. It turns out that establishing the presence of sorting is relatively straightforward since it just requires that more people marry similar rather than different types of individuals to themselves in the dimension we are considering (e.g. education), relative to what would occur if they were matched randomly. Establishing how sorting has changed without reference to a specific model of the marriage market, however, turns out to be difficult and subject to a number of pitfalls. Generally, one would prefer to use a nonparametric approach to measure these changes, that is not mediated by a specific model of the marriage market and not dependent on assumptions about preferences. On the other hand, sorting depends on the structure of the gains from matching with alternative types and changes in sorting depend on how this structure changes.

Constructing a link between the marital surplus, whose structure determines sorting, and the observed matching frequencies will in general depend on the structure of the model assumed to underlie the formation of matches. In this paper we consider alternative approaches to measuring changes in sorting. We will focus on sorting by education level, although the specific characteristic people sort on is not important for our argument; we do consider however sorting only by one single characteristic, which is assumed to have a discrete distribution.

The difficulty in measuring changes in sorting stems from changes in the marginal distributions of education for men and women. This is particularly pertinent because the proportion of people obtaining a college degree has increased substantially over time, differentially for men and women 
so that there are now more women college graduates than men in the US and in other countries (Chiappori et al., 2009).

A first contribution of this paper is to discuss, from a methodological perspective, the way changes in sorting can (and should) be measured. We start by establishing two intuitive criteria that all measures should satisfy if they are to meaningfully measure changes in sorting. We then propose a new index of assortativeness that explicitly links observed marital patterns to the underlying forces driving sorting in marriage, defined by the value of two people marrying each other relative to marrying someone else or remaining single.

Building upon previous contributions by Choo and Siow (2006) and Chiappori et al. (2017) (see also Dupuy and Galichon (2014), Chiappori et al. (2018), Chiappori et al. (2020) and Ciscato and Weber (2020)), we develop the empirical version of this measure in a matching model under the separable extreme value assumption (SEV). We call it the 'SEV index'. The SEV model provides a direct link between the deterministic component of marital surplus and the observed marital frequencies. Positive assortative matching occurs when the surplus is supermodular; increases in sorting happen when supermodularity increases, giving stronger incentives to sort. While this measure depends on distributional assumptions on the unobserved heterogeneity term in the SEV model, namely that preferences for type by marital partners are extreme value, it can readily be extended to other distributions (see Galichon and Salanié (2019)). We also propose a general criterion, called Generalized Separability (GS), that extends the previous approaches by imposing that the assortativeness ranking remains constant for all distributions of unobserved heterogeneity. We provide a full characterization of this criterion. The cost of this general nonparametric approach is that it does not rely on a particular index; in particular, the order relationship it provides on sorting patterns is not complete, in the sense that there exist alternative patterns that cannot be ranked. Thus some ambiguity may remain about whether sorting has changed or not.

The SEV index, as well as its possible extensions and the GS criteria, satisfy our two intuitive criteria. We show that the same property holds for almost all indices used in the literature, such as the standard correlation approach adopted by Greenwood et al. (2003, 2014) or its equivalent $\chi^{2}$ version, the minimum distance criterion introduced by Fernández and Rogerson (2001) and recently used by Abbott et al. (2019) and the weighted sum criterion of Eika et al. (2019). One exception, however, is a likelihood index also introduced in the latter article, which violates one of them. We 
discuss how such a violation may generate spurious conclusions when comparing assortativeness in two populations.

We study changes in assortativeness across cohorts in the US using data from the March extract of the Current Population Survey for the years 1962 to 2019. In a companion paper (Chiappori et al., 2020), we studied assortativeness in the UK using the SEV index. Here we go further, providing an explicit theoretical foundation for that measure, introducing other measures and considering the properties of some existing ways of measuring changes in sorting. We also show results on changes in assortativeness in the US based on a variety of indices, including our SEV index, the generalized version thereof and existing measures from the literature.

Comparing the birth cohorts of the 1930s and 1970s, we find that unambiguous changes in assortativeness, which can be detected by generalised separability approach, are extremely difficult to find in practice. However, both the SEV index and the other measures that have so far been used in the literature show overwhelmingly that marital assortativeness by education increased across the two cohorts. For one single group, that of high-school graduates, we find ambiguous results.

We exploit the structural interpretation of the marital sorting patterns revealed by the SEV approach for counterfactual analysis in order to shed further light on the ways in which assortativeness has changed across the two cohorts. In our counterfactual exercise, we compare observed marital patters for the birth cohort of the 1970s with what they would have been had the value of marriage and assortativeness by education stayed at the 1930s level. We show that the value of marrying declined for all groups with the exception of college educated women, and that the decline was particularly sharp among women with high-school degrees. We also show that changes in the value of different unions between these two cohorts led to higher concentration of marriages in the main diagonal for all education groups except high-school graduates. Moreover, changes in the value of marrying different spouses led to an increase in the proportion of couples where the wife is more educated than the husband and a drop in the the proportion of couples where the husband is more educated.

In the next section we discuss the definition of assortative matching and we introduce the conditions that an index of assortativeness should satisfy if it is to offer a coherent way of measuring changes in sorting. Section 3 introduces the SEV index, which provides a fully structural interpretation of changes in sorting, and section 4 introduces the distribution free generalised criteria. We 
then discuss some alternative measures of sorting that have been used in the literature and investigate their properties in section 5. These include the correlation coefficient and the $\chi^{2}$ criterion, the likelihood criterion and the weighted sum index. Up to this point, our discussion focuses on identifying assortativeness and changes thereof locally, studying separately the sorting between each combination of two types from the distribution of matching traits; this analysis also disregards the possibility of remaining single. In section 6 we discuss how to extend our analysis to consider all marital options as well as singlehood. We then present our empirical results, starting in Section 7 with a brief discussion of the CPS data, followed by the study of how assortativeness changed between the birth cohorts of the 1930s and 1970s in Sections 8 and 9. Section 10 shows how sorting patterns would have been for the 1970s cohort had the value of marriage not changed from the 1930s. A final section concludes.

\section{Assortative matching: an introductory example}

While the concept of assortative matching is reasonably clear, comparing two different matching distributions leads to unexpected difficulties when the marginal distributions of the characteristic upon which people match change over time. Trivially, if there are are many more college graduates now than in the past we are very likely to end up with an increasing number of couples where both are college graduates, unless of course the incentives to remain single change dramatically. However, deciding whether sorting has increased, while accounting for differences in the marginals is less than obvious. We will start with a very simple example to illustrate the difficulties with measuring changes in sorting.

Consider an economy where there is an equal mass of men and women, each normalized to 1. We will abstract from singles at this point - everybody gets matched. The matching pattern in this population is summarized by Table 1 .

Table 1: $2 \times 2$ Matching Table $(m, n, r)$

\begin{tabular}{|c|c|c|}
\hline$w \backslash^{h}$ & $\mathrm{C}$ & $\mathrm{HS}$ \\
\hline $\mathrm{C}$ & $r$ & $m-r$ \\
\hline $\mathrm{HS}$ & $n-r$ & $1-n-m+r$ \\
\hline
\end{tabular}


In the Table, $n$ and $m$ are the proportion of male and female college graduates respectively, while $r$ are the proportion of couples where both spouses are college graduates. In practice, such a matching table is defined by the three numbers $(m, n, r)$; we shall refer to it as 'Table $(m, n, r)$ '. Note that the numbers are non negative and must satisfy:

$$
\max (0, m+n-1) \leq r \leq \min (m, n)
$$

As in this example, in most of our analysis we will consider matching based on only two types of men and two types of women, such as education with two levels: college graduates (C) and less than college (HS).

The general case (involving any number of categories, as well as singles) will be discussed later on. Note, however, that assortativeness is in general a local phenomenon. For instance, it is easy it is easy to provide examples where AM is positive (or increasing) at one end of the distribution while negative (or decreasing) at the other end. This implies that assortativeness measures will have to be local, in the sense that they focus on the marital patterns between two groups and involve considering a (series of) $2 \times 2$ case(s) similar to Table $(m, n, r)$. The term 'local' here does not mean that only adjacent groups in terms of the matching trait can be considered; indeed one can study marital sorting between, say, college graduates and high school dropouts using the same tools we develop here for the analysis of marital patterns among college and high school graduates.

\subsection{Defining Positive Assortative Matching}

We say that a Table T exhibits Positive Assortative Matching (PAM) if the proportion of couples with equal education (the 'diagonal' of the Table) is larger than what would obtain under random matching. Under random matching the proportion of couples where both are college graduates (respectively less than college) will simply be $m n$ (respectively $(1-n)(1-m)$ ), which implies that the Table would become:

Then we have PAM if and only if

$$
r \geq m n
$$


Table 2: Random Matching

\begin{tabular}{|c|c|c|}
\hline$w \backslash^{h}$ & $\mathrm{C}$ & $\mathrm{HS}$ \\
\hline $\mathrm{C}$ & $m n$ & $m(1-n)$ \\
\hline $\mathrm{HS}$ & $n(1-m)$ & $(1-n)(1-m)$ \\
\hline
\end{tabular}

Table $(m, n, m n)$ : Random Matching.

which also implies that:

$$
1-n-m+r \geq(1-n)(1-m)
$$

In other words PAM arises when extra forces generate more matches between equally educated people than would happen for other random reasons.

Now consider two Tables $T_{1}=(m, n, r)$ and $T_{2}=(\bar{m}, \bar{n}, \bar{r})$. Under what conditions can we conclude that say $T_{1}$ is more assortative than $T_{2}$ ? We start with two specific situations in which there exists a natural answer - i.e., in which one can unambiguously assert that a Table displays more AM than another. This will lead to the definition of two Properties; we submit that any acceptable criterion should satisfy these properties.

\subsection{Defining 'increases in Assortative Matching': two required properties}

The case of constant proportions of educated men and women. The first case obtains when comparing two Tables $(m, n, r)$ and $(\bar{m}, \bar{n}, \bar{r})$ that have identical marginal distributions - i.e., the same proportion of educated men and the same proportion of educated women, in which case $m=\bar{m}$ and $n=\bar{n}$. Then the first natural property is the following:

Property 1 (Monotonicity) With $m$ and $n$ fixed (i.e. when the marginal distributions do not change), an increase in $A M$ is equivalent to an increase in $r$ (i.e., more people in each diagonal cell)

In other words, Table $(m, n, r)$ exhibits more AM than Table $(m, n, \bar{r})$ if and only if

$$
r \geq \bar{r}
$$

which also implies that:

$$
1-n-m+r \geq 1-n-m+\bar{r}
$$


The motivation for this property is straightforward: if $m=\bar{m}$ and $n=\bar{n}$ then $r \geq \bar{r}$ means that, for any level of education, a larger fraction of individuals marry their own type in Table $(m, n, r)$ than in Table $(m, n, \bar{r})$. This is the exact definition of increased assortative matching.

The case of perfect sorting. A second polar case obtains when the entire population is concentrated on the diagonal - i.e., $m=n=r$, corresponding to Table $(m, m, m)$ :

Table 3: Perfect Assortative Matching

\begin{tabular}{|c|c|c|}
\hline$w \backslash^{h}$ & $\mathrm{C}$ & $\mathrm{HS}$ \\
\hline $\mathrm{C}$ & $m$ & 0 \\
\hline $\mathrm{HS}$ & 0 & $1-m$ \\
\hline
\end{tabular}

In that case, the matching is perfectly (positive) assortative: all individuals marry a spouse with the same education and there are no 'cross-marriages'. Intuitively, matching cannot possibly be more assortative than in a Table such as $(m, m, m)$. This leads to a second natural property:

Property 2 (Perfectly Assortative Matching) For all possible proportions of educated women $m \in[0,1]$, no Table can display strictly more AM than Table $(m, m, m)$.

\subsubsection{Formal requirements}

We can use these two criteria to define the properties that any index of sorting should satisfy. Thus we now propose that any acceptable criterion for 'increased AM' should be compatible with these two properties. That is, any reasonable criterion should be such that:

- When comparing two Tables with identical marginals, it should boil down to: 'A Table displays more AM if and only if it has more people on the diagonal'.

- A Table in which the entire population is concentrated on the diagonal displays the maximum level of AM.

A formal translation would be the following. We define a matching table as a $2 \times 2$ matrix, the coefficients of which are non-negative and add up to 1, with each cell giving the frequency of occurrence of that match combination; as mentioned above, such a table can be fully summarized 
by three numbers $(m, n, r)$. Our goal is to define a preorder $\succeq_{A M}$ on the set of matching tables, where $(m, n, r) \succeq_{A M}(\bar{m}, \bar{n}, \bar{r})$ reads as ' $(m, n, r)$ displays more AM than $(\bar{m}, \bar{n}, \bar{r})$ '. According to the previous discussion, the preorder must satisfy two conditions:

1. (Monotonicity) For all $(m, n, r, \bar{r})$,

$$
(m, n, r) \succeq_{A M}(m, n, \bar{r}) \text { if and only if } r \geq \bar{r}
$$

2. (Perfectly Assortative Matching) For all $m,(m, m, m)$ is a maximal element of the preorder

A preorder satisfying the two criteria need not be complete, in the sense that there may exist tables that cannot be ranked using the preorder. In particular, condition 2 does not imply that $(m, m, m)$ displays more AM than any table, but only that no table can display strictly more AM than $(m, m, m)$. However, some criteria commonly used in the literature rely on an index of assortativeness $I(m, n, r)$ that quantifies the 'degree of assortativeness' as a function of $m, n$ and $r$. In that case, the preorder relationship is implied by this index:

$$
(m, n, r) \succeq_{A M}(\bar{m}, \bar{n}, \bar{r}) \text { if and only if } I(m, n, r) \geq I(\bar{m}, \bar{n}, \bar{r})
$$

and is complete. But the question is whether it satisfies our two conditions. Specifically, condition 1 requires that $I(m, n, r)$ be increasing in $r$, while condition 2 requires:

$$
I(m, m, m) \geq I(\bar{m}, \bar{n}, \bar{r}) \text { for all }(m, \bar{m}, \bar{n}, \bar{r})
$$

We now describe some of the criteria that have been used most frequently in the literature. We start with a specific family of indices that are based on a structural interpretation, following the seminal approach by Choo and Siow (2006); next, we discuss several alternative indices.

\section{The Separable Extreme Value (SEV) approach and its extensions}

Starting with Becker (1981) it is well understood that sorting in the marriage market arises as a consequence of complementarities between the characteristics of the partners in the value function 
of the household. Building on this idea the seminal contribution of Choo and Siow (2006) offers a structural interpretation of assortative matching in a context where cross marriages can and do occur. Their approach ignores search or other frictions, and relates sorting to the underlying force that drives matching, namely the properties of the surplus of the match, which reflects the gains from two people with certain characteristics matching together instead of partnering with alternative types or perhaps remaining single. The framework is general enough to allow matching on both observable and unobservable characteristics and to allow for idiosyncratic preferences for types of spouses. The model is similar in structure to random utility models such as the one presented in Berry et al. (1995).

The demands of men and women need to be made consistent. Given preferences and the structure of the surplus, the equilibrium is achieved by setting the share of the surplus enjoyed by men and women in each type of match (say HS,C) so that male and female demands are made consistent. In the equilibrium that results - a stable matching equilibrium - no person matched would rather be single and no two people not matched to each other would rather be together than remaining in their current situation. In a transferable utility context this equilibrium maximizes social welfare.

Changes in sorting are linked directly to changes in the structure of the surplus: if this becomes more supermodular, meaning that the complementarities are stronger, then there will be more positive sorting. The key point is that the SEV model, given distributional assumptions on preferences provides a direct link between changes in the matching probabilities and changes in the extent to which the surplus is supermodular. We now use these ideas to derive explicitly a criterion that will allow us to rank alternative matching tables.

\subsection{The basic framework}

\subsubsection{Frictionless matching under TU}

In the SEV model, individuals play a frictionless, bipartite matching game under Transferable Utility (TU). ${ }^{1}$ In such a game, men $X$ and women $Y$ may either remain single or match with a member of the opposite sex. A match between Ms. $i$ and Mr. $j$ generates a gain $s_{i j}$ that must be allocated between spouses; and the TU assumption requires that the utility of Ms. $i, u_{i}$, and the utility of

\footnotetext{
${ }^{1}$ See Chiappori (2017) for a precise description of frictionless matching models.
} 
Mr. $j, v_{j}$, must add up to that gain:

$$
u_{i}+v_{j}=s_{i j}
$$

The matching game is fully defined by the two sets $X$ and $Y$, endowed each with a finite measure, and the gain $s_{i j}$ for each potential pair $(i \in X, j \in Y)$. In general, singles' utilities are normalized to zero; then $s_{i j}$ can be interpreted as a surplus representing the total benefit generated by the match over and above what $i$ and $j$ could obtain as singles.

Finally, a matching thus defined is stable if (i) no matched individual would be better off as single, and (ii) no pair $(i, j)$ would both prefer being matched together better than their current situation (the latter condition may be interpreted as 'divorce at will').

\subsubsection{The SEV model}

Starting from this framework, the SEV model relies on several additional assumptions:

1. The sets $X$ and $Y$ include a 'large' number of people but these people can be classified into a 'small' number of observable classes, $I \in\{1, \ldots, N\}$; in what follows, these will be interpreted for clarity as education levels.

2. The surplus generated by the matching of $i \in I$ and $j \in J$ is the sum of two components:

$$
s_{i j}=Z^{I J}+\gamma_{i j}
$$

where $Z^{I J}$ is a deterministic component depending only on the individuals' education classes, and $\gamma_{i j}$ is a random term reflecting unobserved heterogeneity to be interpreted as the preference that the individuals have for each other beyond the economic value of the match that is driven by the observable characteristics. A natural (although not exclusive) interpretation is that $\gamma_{i j}$ reflects the impact of some traits that influence the gain generated by the match but are not observed by the econometrician. Similarly, the utility of Ms. $i$ (resp. Mr. j) as single (denoted by $\emptyset)$ is

$$
s_{i \emptyset}=\varepsilon_{i}^{\emptyset} \text { and } s_{\emptyset j}=\nu_{j}^{\emptyset}
$$

Under an adequate normalization (which amounts to imposing that the deterministic part of 
the surplus of singles is always nil), the matrix $Z=\left(\left[Z^{I J}\right]\right)$ thus represents the systematic influence of education on matching patterns, whereas all other effects (including idiosyncratic components) are summarized by the random terms $\left(\varepsilon_{i}^{\emptyset}, \nu_{j}^{\emptyset}, \gamma_{i j}\right)$.

The 'supermodular cores' of matrix $\mathrm{Z}$ are defined, for all $I<K$ and $J<L$, as the double difference:

$$
S=Z_{I J}+Z_{K L}-Z_{I L}-Z_{K J}
$$

As we shall see, these play a key role in the characterization of assortativeness.

3. The random term $\gamma_{i j}$ is separable $(\mathrm{S})$ :

$$
\gamma_{i j}=\varepsilon_{i}^{J}+\nu_{j}^{I}
$$

where $\varepsilon_{i}^{J}$ and $\nu_{j}^{I}$ are individual-specific random terms that exclusively depend on the partner's education. Intuitively, $\varepsilon_{i}^{J}$ can be interpreted either as an unobservable trait of Ms. $i$ which is valued differently by spouses with different educations, or as Ms. i's idiosyncratic (and unobservable) preference for her spouse's education.

4. Finally, SEV assume that the $\varepsilon \mathrm{s}$ and the $\nu$ s are independent and type 1 extreme value (EV),

implying that the differences $\varepsilon_{i}^{J}-\varepsilon_{i}^{K}$ and $\nu_{j}^{I}-\nu_{j}^{K}$ follow a logistic distribution for all $i, j$ and all $I, J, K$.

\subsubsection{Properties}

Under these assumptions, several results can be derived. The first one provides an exact characterization of individual utilities.

Proposition 1 (Chiappori et al., 2017; Choo and Siow, 2006) Under assumptions 1, 2 and 3 above, there exists $2 \times N^{2}$ deterministic values $U^{I J}, V^{I J}, I, J=1, \ldots, N$, with $Z^{I J}=U^{I J}+V^{I J}$ for all $(I, J)$, such that for any matched pair $(i, j)$ at a stable matching, with $i \in I$ and $j \in J$, individual utilities are equal to:

$$
u_{i}=U^{I J}+\varepsilon_{i}^{J} \text { and } v_{j}=V^{I J}+\nu_{j}^{I}
$$


The significance of this result is that each individual benefits from their own unobserved preference for type of partner, and does not share this. Only the deterministic component is shared, which also drives the extent of sorting.

A second property is that the SEV model is exactly identified from matching patterns. Here, matching patterns are fully described by a set $M=\left(\mu^{I J} I, J=\emptyset, 1, \ldots, N\right)$ where $\mu^{I J}$ denotes the fraction of couples where the wife has education $I$ and the husband has education $J$, and $\mu^{I \emptyset}$ (resp. $\left.\mu^{\emptyset J}\right)$ the fraction of single women (men) with education $I(J)$ and $^{2}$

$$
\sum_{I, J} \mu^{I J}=1
$$

Then we have the following result which defines the relationship between the observed matching patterns (who marries whom) and the value of that match that accrues to each partner. This result is the same as the one underlying McFadden's conditional choice probabilities in discrete choice problems (McFadden, 1981).

Proposition 2 (Choo and Siow, 2006) Under assumptions 1, 2, 3 and 4 above, for any $M$ there exist exactly one vector $Z=\left(Z^{I J} I, J=1, \ldots, N\right)$ such that the probability that, at a stable matching, a woman $i \in I$ has a husband in $J$ (resp. is single) is $\mu^{I J} / \sum_{K} \mu^{I K}$ (resp. $\mu^{I \emptyset} / \sum_{K} \mu^{I K}$ ) and the probability that, at a stable matching, a man $j \in J$ has a wife in $I$ (resp. is single) is $\mu^{I J} / \sum_{K} \mu^{K J}\left(\operatorname{resp} . \mu^{\emptyset J} / \sum_{K} \mu^{K J}\right)$.

The intuition underlying this result is simple. Note, first, that by Proposition 1, Ms. $i \in I$ will marry a husband in class $J$ if and only if

$$
U^{I J}+\varepsilon_{i}^{J} \geq U^{I K}+\varepsilon_{i}^{K} \text { for all } K
$$

This is a standard discrete choice model, where the $\varepsilon \mathrm{s}$ are moreover extreme value distributed. It follows that a multi-logistic regression will exactly identify the $U^{I J}$ for all $J$ (under the normalization $\left.U^{I \emptyset}=0\right)$. The same is true for all education levels $I$; and the $V^{I J}$ can similarly be recovered from multi-logistic regressions of husband's marital choices. Finally, $Z^{I J}=U^{I J}+V^{I J}$ for all $(I, J)$.

\footnotetext{
${ }^{2}$ Under the normalizations $\mu^{\emptyset \emptyset}=0$
} 


\subsection{The SEV assortativeness index for two classes of people}

\subsubsection{Characterization}

The previous results directly apply to the $2 \times 2$ examples discussed in the previous section. For the time being, we closely follow the spirit of these examples by omitting singles and concentrating on matching patterns among married couples. As a result, the uniqueness of the structural matrix $\left(\left[Z^{I J}\right]\right)$ no longer obtains: different structural matrices may generate identical matching patterns among married couples, although they produce different percentages of singles. However, this indeterminacy is irrelevant for the analysis of assortativeness.

Formally:

Proposition 3 Table $(m, n, r)$ can be generated by any SEV model such that its supermodular core satisfies

$$
Z^{11}+Z^{22}-Z^{12}-Z^{21}=2 \ln \left(\frac{r(1+r-m-n)}{(n-r)(m-r)}\right)
$$

In particular, one of the structural matrices that would generate Table $(m, n, r)$ is simply:

$$
Z=2\left(\begin{array}{cc}
\ln r & \ln (m-r) \\
\ln (n-r) & \ln (1+r-m-n)
\end{array}\right)
$$

Proof. In the appendix

In the $2 \times 2$ case, the the supermodular core of the matrix $\mathrm{Z}$ is unique. As mentioned above, it plays a crucial role in the analysis of assortativeness. A standard theoretical result of the matching literature is that assortative matching is related to supermodularity of the surplus function. We now proceed to show that this intuition extends to our context: supermodularity of the deterministic ('structural') part of the surplus (the $Z \mathrm{~s}$ ), which is equivalent to its supermodular core being positive, directly translates into assortativeness of matching patterns. Specifically, let us first define the $S E V$ assortativeness index $I_{S E V}$ :

Definition 4 The SEV assortativeness index $I_{S E V}$ corresponding to Table $(m, n, r)$ is defined by:

$$
I_{S E V}(m, n, r)=\ln \left(\frac{r(1+r-m-n)}{(n-r)(m-r)}\right)
$$


In words, the index is the $\log$ of a ratio, the numerator of which is equal to the product of diagonal cells while the denominator is the product of off-diagonal cells.

This index satisfies the two required key properties:

Proposition 5 The index $I_{S E V}(m, n, r)$ :

1. is increasing in $r$ (for $m$ and $n$ constant) and equal to 0 for $r=m n$. In particular, $I_{S E V}(m, n, r) \geq$ 0 if and only if $r \geq m n$

2. goes to $+\infty$ when either $r \rightarrow m$ or $r \rightarrow n$

These properties, in turn, have direct economic interpretations. To see how, note, first, that the index equals zero under random matching (if $r=m n$ ), and is positive if and only if the Table exhibits PAM. Secondly, the supermodular core of the deterministic ('structural') part of the surplus is:

$$
Z^{11}+Z^{22}-Z^{12}-Z^{21}=2 I_{S E V}(m, n, r)
$$

In particular, the matrix $Z$ is supermodular if and only if $I_{S E V}(m, n, r) \geq 0$. It follows that Table $(m, n, r)$ displays PAM (defined as $r \geq m n)$ if and only if $I_{S E V}(m, n, r) \geq 0$ - i.e., if and only if the structural matrix $Z$ is supermodular.

Finally, for the matrix $(m, m, m)$ (which corresponds to Perfectly Assortative Matching), we have $I_{S E V}(m, m, m)=+\infty$; therefore the index $I_{S E V}(m, n, r)$ satisfies the second requirement of Section $4 .^{3}$

\subsubsection{Comparing Assortative Matching}

The previous results suggest a first criterion for comparing Assortative Matching (AM) across tables:

Definition 6 Table $(m, n, r)$ displays more AM than Table $(\bar{m}, \bar{n}, \bar{r})$ in the SEV sense if and only if

$$
I_{S E V}(m, n, r) \geq I_{S E V}(\bar{m}, \bar{n}, \bar{r})
$$

From the previous results, an equivalent definition is the following:

\footnotetext{
${ }^{3}$ In fact, the index satisfies an even stronger property: $\Phi_{C S}(m, n, r)=+\infty$ whenever $r=\min (m, n)$, so that the index is maximal as soon as one off-diagonal element of the Table is equal to zero.
} 
Definition 7 Table $(m, n, r)$ displays more AM than Table $(\bar{m}, \bar{n}, \bar{r})$ in the SEV sense if and only if the structural matrix corresponding to $(m, n, r)$ is more supermodular than that corresponding to $(\bar{m}, \bar{n}, \bar{r})$, in the sense that

$$
Z^{11}+Z^{22}-Z^{12}-Z^{21} \geq \bar{Z}^{11}+\bar{Z}^{22}-\bar{Z}^{12}-\bar{Z}^{21}
$$

In other words, a given Table displays more AM than another if and only if the supermodular core of its structural matrix is larger. By Proposition 3, there is a one-to-one correspondence between a table and the supermodular core of its structural matrix, which proves that the definition is consistent.

The SEV criterion has (implicitly) been used in several contributions, among which Choo and Siow (2006) and Chiappori et al. (2017). The main advantage of this definition is that it is directly related to a structural model; in particular, it creates a natural link between the empirical notion of 'assortativeness' and its theoretical interpretation in terms of supermodularity of the surplus function. The separability assumption (2) requires in particular that the stochastic terms, which reflects unobserved heterogeneity, do not introduce any super- (or sub-) modularity; thus the model imposes that assortativeness be directly related to supermodularity of the deterministic component. The disadvantage is that it depends on specific assumptions on the distribution of preferences, which in this context at least cannot be tested. We discuss in the next section how the results can be sensitive to these assumptions, and how a distribution free criterion can be constructed.

\subsection{Extensions of SEV: relaxing the extreme value assumption}

A recent literature (Galichon and Salanié, 2019) has relaxed the last assumption of the standard SEV model (assumption 4 in subsection 3.1.2). Indeed, while assuming extreme value distributions may seem natural in this context, it is by no means necessary; in fact, any (known) distributions can be used. Moreover, the main conclusions of the previous section remain valid for any specific distribution. Proposition 1 does not require the extreme value assumption to hold; and one can readily show that, if the distribution of the random terms is known ex ante, then there is a one-toone correspondence between the marital patterns (including singles) and the underlying structural matrix whatever the particular (known) distributions. 
Of particular importance in practice is the relationship between supermodularity of the structural part of the surplus and assortativeness of matching patterns. Indeed, a result by Graham (2011, 2013), further generalized by Chiappori (2017), establishes that supermodularity of the deterministic part of the surplus (the $Z \mathrm{~s}$ ) directly translates into assortativeness of the matching patterns, irrespective of the distributions of the random terms. Specifically, consider the matching tables generated by two models with the same distributions of the random terms $\varepsilon \mathrm{s}$ and $\nu \mathrm{s}$ and the same marginals $(m=\bar{m}$ and $n=\bar{n}$ ) but different structural matrices, say $Z$ and $\bar{Z}$. Then the matching table generated by $Z$ displays more AM that that generated by $\bar{Z}$ (in the sense, discussed above, that the former has more people on the diagonal that the latter) if and only if $Z$ is more supermodular than $\bar{Z}$.

Formally, we replace assumption 4 of subsection 3.1 .2 by the following:

Assumption 4' The individual random vectors $\left(\varepsilon_{i}^{1}, \varepsilon_{i}^{2}\right)$ (resp. $\left.\left(\nu_{j}^{1}, \nu_{j}^{2}\right)\right)$ are drawn from some distribution $F_{\varepsilon}\left(\right.$ resp. $\left.G_{\nu}\right)$ on $R^{2}$. They are independent of each other and independent of the individual's education; moreover, the distribution of $\left(\varepsilon_{i}^{1}-\varepsilon_{i}^{2}\right)\left(\right.$ resp. $\left.\left(\nu_{j}^{1}-\nu_{j}^{2}\right)\right)$ is symmetric.

Note that we do not assume that $\varepsilon_{i}^{1}$ and $\varepsilon_{i}^{2}$ (or $\nu_{j}^{1}$ and $\nu_{j}^{2}$ ) are independent from each other the distributions $F_{\varepsilon}$ and $G_{\nu}$ can display any correlation structure; nor do we suppose that the two distributions are identical.

The result is the following:

Proposition 8 (Chiappori, 2017; Graham, 2011, 2013) Consider two matching Tables (m, n, r) and $(\bar{m}, \bar{n}, \bar{r})$, generated by two models that both satisfy assumptions 1, 2, 3 and 4'. Assume that the random distributions $F_{\varepsilon}$ and $F_{\nu}$ are the same in the two models, while the structural matrices $Z$ and $\bar{Z}$ are different. Assume, moreover, that $m=\bar{m}$ and $n=\bar{n}$. Then $r \geq \bar{r}$ if and only if $Z$ is more supermodular than $\bar{Z}$ :

$$
Z^{11}-Z^{12}-Z^{21}+Z^{22} \geq \bar{Z}^{11}-\bar{Z}^{12}-\bar{Z}^{21}+\bar{Z}^{22}
$$

Proof. See Appendix

The result by Graham (2011) states that, in a model of this type, the matching patterns display PAM (i.e. more people are on the diagonal than would obtain under random matching) if and 
only if the supermodular core of the corresponding deterministic structure is positive. In addition, proposition 8 allows the comparison of any two tables according to the same criterion so long as the marginal distribution of the characteristics are the same, i.e. in our example the proportion of men and women in each education group are the same in each table. Since zero supermodularity (defined by $Z^{11}+Z^{22}-Z^{12}-Z^{21}=0$ ) gives random matching, Proposition 8 implies Graham's result.

The economic implication of Proposition 8 is clear. If we apply a separable approach with arbitrary distributions for the random term, it remains true that, when comparing two matching tables with identical marginals, a table displays more AM (i.e., it has more people on the diagonal) than the other if and only if its structural matrix is more supermodular.

This suggests the following definition:

Definition 9 For any given distributions of the $\varepsilon s$ and $\nu$ s satisfying Assumption 4', we say that table $(m, n, r)$ displays more $A M$ than $(\bar{m}, \bar{n}, \bar{r})$ for these distributions if and only if, for these distributions, the supermodular core associated to $(m, n, r)$ is larger than the supermodular core associated to $(\bar{m}, \bar{n}, \bar{r})$ :

$$
Z^{11}+Z^{22}-Z^{12}-Z^{21} \geq \bar{Z}^{11}+\bar{Z}^{22}-\bar{Z}^{12}-\bar{Z}^{21}
$$

It is crucial to note at this point that, for a given matching table, the estimation of the supermodular core of the structural component depends on the random distributions. In other words, for any specific choice of the distributions, marital patterns exactly determine the supermodular core of the underlying, structural matrix; but this correspondance varies with the distributions at stake.

\section{The Generalized Separable (GS) approach}

\subsection{Definition}

We have seen in Subsection 3.3 that a separable approach could use pretty much any distribution for the stochastic terms. That fact, however, points to a clear weakness of the SEV approach, because its conclusions regarding variations in assortativeness typically depend on the distribution of the random term. We may empirically find that Table $(m, n, r)$ displays more AM than Table $(\bar{m}, \bar{n}, \bar{r})$, 
but this conclusion remains contingent on the specific choice of the distributions of the random term - i.e., in the standard SEV framework, on the extreme value assumption. This is particularly unsatisfactory because these distributions cannot be empirically identified from the sole observation of matching patterns, but have to be assumed ex ante. The conclusion thus partly relies on the arbitrary choice of the distribution. In particular, it may well be the case that, when comparing two Tables using an extended separable model, one Table displays more PAM than the other for some choices of the random distributions of the $\varepsilon \mathrm{s}$ and $\nu \mathrm{s}$, while the opposite is true for other distributions.

Assume, however, that the tables to be compared are such that a separable criterion would conclude that one displays more AM (in the sense of Definition 9) for all possible distributions satisfying Assumption 4'; then one could reliably conclude that AM has increased.

This suggests the following Definition:

Definition 10 We say that Table $(m, n, r)$ displays more AM than Table $(\bar{m}, \bar{n}, \bar{r})$ in the GS sense:

$$
(m, n, r) \succeq_{G S}(\bar{m}, \bar{n}, \bar{r})
$$

if and only if, for any choice of the distributions of the $\varepsilon s$ and $\nu$ s satisfying Assumption 4', Table $(m, n, r)$ displays more AM than Table $(\bar{m}, \bar{n}, \bar{r})$ for these distributions.

From a formal perspective, this criterion defines a preorder relationship $\succeq_{G S}$ on the set of matching tables. Importantly, this preorder is not complete; indeed, Table $(m, n, r)$ may display more AM than $(\bar{m}, \bar{n}, \bar{r})$ for some distributions but less for others, in which case the two Tables cannot be compared in the GS sense.

\subsection{Characterization}

We now provide a simple and tractable characterization of the previous definition.

Proposition 11 Under the same assumptions as for Proposition 8, assume that Table $(m, n, r)$ and $(\bar{m}, \bar{n}, \bar{r})$, which both display PAM, are such that:

$$
\frac{r}{m} \geq \frac{\bar{r}}{\bar{m}}, \frac{r}{n} \geq \frac{\bar{r}}{\bar{n}}, \quad \frac{1-n-m+r}{1-n} \geq \frac{1-\bar{n}-\bar{m}+\bar{r}}{1-\bar{n}}
$$




$$
\text { and } \quad \frac{1-n-m+r}{1-m} \geq \frac{1-\bar{n}-\bar{m}+\bar{r}}{1-\bar{m}}
$$

Then $(m, n, r)$ displays more AM than $(\bar{m}, \bar{n}, \bar{r})$ in the $G S$ sense.

Conversely, if one of these inequalities is violated, then it is possible to find random distributions such that $(\bar{m}, \bar{n}, \bar{r})$ displays more $A M$ than $(m, n, r)$ for these distributions.

Proof. See Appendix

The inequalities in Proposition 11 have a simple interpretation; namely, the proportion of each diagonal term increases within the corresponding row and column. If that's the case, then for any choice of stochastic distributions, the surplus corresponding to $(m, n, r)$ will be more supermodular than the surplus corresponding to $(\bar{m}, \bar{n}, \bar{r})$. Moreover, the condition is also necessary: if one of these inequalities is violated, then one can find distributions such that the conclusion is reversed.

One can easily show that the GS preorder satisfies the two Properties described in Subsection 4. Proposition 8 shows that the first condition is satisfied. Regarding the second, assume that we have

$$
(\bar{m}, \bar{n}, \bar{r}) \succ_{G S}(m, m, m)
$$

for some $m, \bar{m}, \bar{n}, \bar{r}$. By Proposition 11, this requires that at least one of the four ratios in Proposition 11 is strictly larger for $(\bar{m}, \bar{n}, \bar{r})$ than for $(m, m, m)$. But this is impossible, since these ratios are all less than or equal to 1 by construction and they are all equal to 1 for $(m, m, m) .{ }^{4}$ Incidentally, this argument shows that Proposition 11 implies a stronger version of the Perfectly Assortative Matching condition: not only is $(m, m, m)$ an extremal element, but it directly dominates any other Table. That is, although the preorder $\succeq_{G S}$ is not complete, we have:

$$
(m, m, m) \succeq_{G S}(\bar{m}, \bar{n}, \bar{r})
$$

for all $m, \bar{m}, \bar{n}, \bar{r}$ :

\footnotetext{
${ }^{4}$ Alternatively, one cannot find $(\bar{m}, \bar{n}, \bar{r})$ that displays more AM than $(m, n, r)$ for all distributions, since that would hold in particular for the extreme value distributions used in the SEV approach; but that is impossible since $\Phi_{S E V}(m, m, m)=+\infty$.
} 


\section{$5 \quad$ Alternative measures of Assortativeness}

There have been a number of approaches to measure assortativeness in the literature, that are not necessarily based on some underlying structural model, at least explicitly. We now examine some common or influential measures, based on the criteria we have developed.

\subsection{Correlation and $\chi^{2}$}

Probably the simplest way of measuring the degree of assortativeness is to compute the correlation between husband's and wife's educations. This has been used in various contributions (for instance Greenwood et al., 2003, 2014), either explicitly or through a linear regression framework. ${ }^{5}$

In our $2 \times 2$ setting, we can consider female (resp. male) education as a Bernoulli random variable taking the value $E$ with probability $m$ (resp. $n$ ). Then the correlation is simply:

$$
I_{\text {Corr }}(m, n, r)=\frac{r-m n}{\sqrt{m n(1-m)(1-n)}}
$$

which leads to the following definition:

Definition 12 A Table $(m, n, r)$ displays more $A M$ than $(\bar{m}, \bar{n}, \bar{r})$ in the correlation sense if and only if

$$
I_{\text {Corr }}(m, n, r) \geq I_{C o r r}(\bar{m}, \bar{n}, \bar{r})
$$

In other words, according to that criterion, one can state that AM has increased if and only if the correlation between spouses' educations has increased. One can readily check that the two Properties described in Subsection 4 are satisfied. The correlation is nil for $r=m n$ and increasing in $r$; it follows that when comparing tables with the same marginals, the table with more people on the diagonal will have a larger correlation. Moreover, if $m=n=r$ then $I_{\text {Corr }}(m, m, m)=1$, which

\footnotetext{
${ }^{5}$ An abundant sociological literature, starting with Mare (1991) and including Schwartz and Mare (2005), consider the so-called 'log-linear model', based on regressions of the $(\log )$ size of each cell on the $(\log )$ size of the corresponding row and column. This approach, however, generates methodological problems. For one thing, while the various regressions should satisfy adding up restrictions (since the cell sizes of a given row add up to the size of the row), no simple condition on the corresponding regression parameters can guarantee that these properties are satisfied. Moreover, whether such a 'matching function' can at all be deduced from a structural matching model is not clear; for instance, in any structural model the number of people in a given cell would depend on the size of all rows and columns, not only on the size of that cell's row and column. See for instance chapter 4 in Chiappori (2017) for a detailed discussion.
} 
is the maximum value the correlation can take, which proves that the criterion satisfies the Perfect Assortative Matching condition.

One can, equivalently, consider the $\chi^{2}$ distance between observed matching patterns and what would obtain under random matching. For the above Table, the corresponding statistic is just the square of the correlation:

$$
\chi^{2}=\frac{1}{m n} \frac{(r-m n)^{2}}{(1-m)(1-n)}
$$

implying that when comparing Tables that both display PAM, Table $(m, n, r)$ displays more AM than Table $(\bar{m}, \bar{n}, \bar{r})$ in the correlation sense if and only if it displays more AM than Table $(\bar{m}, \bar{n}, \bar{r})$ in the $\chi^{2}$ sense.

\subsection{The minimum distance approach}

An alternative approach can be found for instance in Fernández and Rogerson (2001) and Abbott et al. (2019). The main idea can be described as follows. Starting from a given matrix, consider two extreme matrices with the same marginals: one corresponds to random matching, the other to perfectly assortative matching. Then one can construct the convex combination of these two extreme cases that minimizes the distance with the initial matrix; the corresponding weight can be used as an indicator of AM, which is equal to zero under random matching and to 1 for perfectly assortative matching.

We illustrate the idea in Table 4 where the top panel shows random matching, the second panel is perfect assortative matching for the case where then are more college educated women than men, and the lowest panel is the convex combination, where $\lambda \in[0,1]$ must be chosen to provide the closest approximation to the actual marriage patterns (here, we assume, without loss of generality, that $m \geq n)$.

Then $\lambda$ is chosen to minimize the distance (in the standard, geometric sense) between this convex combination and the initial matrix; we thus consider the problem:

$$
\begin{aligned}
\min _{\lambda} & {[\lambda n+(1-\lambda) m n-r]^{2}+[\lambda(m-n)+(1-\lambda) m(1-n)-(m-r)]^{2} } \\
& +[(1-\lambda) n(1-m)-(n-r)]^{2} \\
& +[\lambda(1-m)+(1-\lambda)(1-m)(1-n)-(1-n-m+r)]^{2}
\end{aligned}
$$


Table 4: The Fernandez and Rogerson index

\begin{tabular}{|c|c|c|}
\hline \hline \multicolumn{2}{|c|}{ Random Matching } \\
\hline$w \backslash^{h}$ & $\mathrm{C}$ & $\mathrm{HS}$ \\
\hline $\mathrm{C}$ & $m n$ & $m(1-n)$ \\
\hline $\mathrm{HS}$ & $n(1-m)$ & $(1-n)(1-m)$ \\
\hline
\end{tabular}

Perfect Assortative Matching

\begin{tabular}{|c|c|c|}
\hline$w \backslash^{h}$ & $\mathrm{C}$ & $\mathrm{HS}$ \\
\hline $\mathrm{C}$ & $n$ & $m-n$ \\
\hline $\mathrm{HS}$ & 0 & $1-m$ \\
\hline
\end{tabular}

Convex Combination

\begin{tabular}{|c|c|c|}
\hline$w \backslash^{h}$ & $\mathrm{C}$ & $\mathrm{HS}$ \\
\hline $\mathrm{C}$ & $\lambda n+(1-\lambda) m n$ & $\lambda(m-n)+(1-\lambda) m(1-n)$ \\
\hline $\mathrm{HS}$ & $(1-\lambda) n(1-m)$ & $\lambda(1-m)+(1-\lambda)(1-m)(1-n)$ \\
\hline \hline
\end{tabular}

The solution to this minimization problem is the minimum distance index; here, it is equal to:

$$
I_{M D}(m, n, r)=\frac{r-m n}{n-m n}
$$

which satisfies both the monotonicity criterion and the Perfect Assortative Matching condition. This suggests the following definition:

Definition 13 A Table $(m, n, r)$ displays more $A M$ than $(\bar{m}, \bar{n}, \bar{r})$ in the minimum distance sense if and only if

$$
I_{M D}(m, n, r) \geq I_{M D}(\bar{m}, \bar{n}, \bar{r})
$$

\subsubsection{Shen's Perfect-Random Normalization}

In a recent contribution, Shen (2019), exploiting an initial suggestion by Liu and Lu (2006), suggests yet another index, defined by normalizing 'the random matching counterfactual to equal 0 and the perfect matching counterfactual to equal 1' and asking 'where in between these two bounds the observed' (p.14 in Shen (2019)). In other words, the idea is again to compare the existing 
distribution to two benchmarks, namely random and perfectly assortative matchings, by computing for each diagonal cell the ratio:

$$
\frac{\text { observed - random }}{\text { perfect - random }}
$$

Although the criterion independently considers each education level, its value, in our $2 \times 2$ case, is the same for both levels, and equal to (still assuming $m \geq n$ ):

$$
I_{P R N}(m, n, r)=\frac{r-m n}{n-m n}
$$

which exactly coincides with the minimum distance.

\subsection{The likelihood approach}

Recent contributions (for instance Eika et al., 2019) refer to yet another criterion. They measure marital sorting between men of education level $I$ and women of education level $J$ "as the observed probability that a husband with education level I is married to a wife with education level $J$, relative to the probability under random matching with respect to education" (page 2804). This criterion is justified as follows: "positive (negative) educational assortative mating is defined as men and women with the same level of education marrying more (less) frequently than what would be expected under a marriage pattern that is random in terms of education." (p. 2796, footnote 1).

\subsubsection{The likelihood ratios}

In practice, this leads to computing two different ratios. One is specific to each education level; in the $2 \times 2$ case, this approach boils down to using a specific index, namely the likelihood ratio:

$$
I_{L}(m, n, r)=\frac{r}{n m}
$$

The corresponding preorder becomes:

$$
(m, n, r) \succeq_{L}(\bar{m}, \bar{n}, \bar{r}) \text { if and only if } \frac{r}{m n} \geq \frac{\bar{r}}{\bar{m} \bar{n}}
$$


Using this criterion, assortative matching has increased from table $(\bar{m}, \bar{n}, \bar{r})$ to table $(m, n, r)$ if and only if the corresponding ratio is larger for table $(m, n, r)$. Using this criterion, Eika et al. (2019) conclude in particular that, in the US over the last decades, AM has decreased at the top of the distribution by education.

\subsubsection{The weighted sum index}

A second index, also used in Eika et al. (2019), considers the weighted sum of likelihood ratios along the diagonal; the weights, here, may be the probabilities of each education level in either the female or the male marginal distributions of education - or any convex combination of these two marginals. In the $2 \times 2$ case, thus, take any weighting function $\phi(m, n) \in[0,1]$ such that

$$
\min (m, n) \leq \phi(m, n) \leq \max (m, n)
$$

which implies in particular that $\phi(m, m)=m$, and consider the index

$$
I_{L \phi}(m, n, r)=\frac{r}{n m} \times \phi(m, n)+\frac{1+r-m-n}{(1-m)(1-n)} \times(1-\phi(m, n))
$$

For instance, if the weight is simply the fraction of women in each education class, $\phi(m, n)=m$ which gives:

$$
I_{L F}=\frac{r}{n}+\frac{1+r-m-n}{1-n}
$$

while using the fraction of men in each education class, $\phi(m, n)=n$, gives

$$
I_{L M}=\frac{r}{m}+\frac{1+r-m-n}{1-m}
$$

Finally, using the average:

$$
\phi(m, n)=\frac{m+n}{2}
$$

we have that

$$
I_{L A}=\frac{1}{2} I_{L F}+\frac{1}{2} I_{L M}
$$




\subsubsection{Properties}

Interestingly, the properties of these two indices are quite different. Both obviously satisfy the monotonicity Property defined above. The second, Perfect Assortative Matching condition is also satisfied by the weighted index, as stated by the following result

Proposition 14 For any $\phi(m, n) \in[0,1]$ such that $\phi(m, m)=m, I_{L \phi}(m, n, r) \leq 2$. Moreover, $I_{L \phi}(m, m, m)=2$

Proof. Assume for instance that $m \geq n$. Then

$$
I_{L \phi}(m, n, r) \leq \frac{r}{n m} \times n+\frac{1+r-m-n}{(1-m)(1-n)} \times(1-m)=\frac{r}{m}+\frac{1+r-m-n}{1-n}
$$

and since $r \leq n$

$$
I_{L \phi}(m, n, r) \leq\left(\frac{1}{m}+\frac{1}{1-n}\right) n+\frac{1}{n-1}(m+n-1)
$$

The right hand side expression is increasing in $n$ and decreasing in $m$; under $m \geq n$, its maximum obtains for $m=n$, in which case it is equal to 2. Finally, one can readily check that $I_{L \phi}(m, m, m)=$ 2.

On the contrary, the non weighted index obviously violates that condition, as it will become clear from the example in Section 5.5.

\subsection{Comparing the indices}

A natural question, at that point, relates to the relationships that may exist between the various criteria defined so far. In particular, the GS criterion introduced above generalizes the various separable indices that are based on a specific random distribution, in the sense that if Table A displays more AM than Table B in the GS sense, it also does for any separable criterion. Is this conclusion also valid for other indices? In other words, is it true that if Table A displays more AM than Table B in the GS sense, it also does for the correlation (or the $\chi^{2}$ ), the minimum distance and the likelihood indices?

Regarding the correlation $-\chi^{2}$ criterion, a positive answer is given by the following result: 
Proposition 15 If a change in $(m, n, r)$ increases $A M$ in the GS sense then it must increase the $\chi^{2}$ statistic. However, the converse is false: one can find changes that increase the $\chi^{2}$ but do not increase AM in the GS sense, implying that they decrease AM for some specific distributions of the random terms.

Proof. See Appendix.

A similar result obtains for the minimum distance criterion:

Proposition 16 If a change in $(m, n, r)$ increases $A M$ in the GS sense then it must increase the minimum distance index. However, the converse is false: one can find changes that increase the minimum distance index but do not increase $A M$ in the GS sense, implying that they decrease $A M$ for some specific distributions of the random terms

Proof. See Appendix.

We now consider the Likelihood criterion. We know that for the simple unweighted likelihood index, the answer must be negative, since this index does not satisfy the Perfect Assortative Matching condition: any perfectly assortative matching is a maximum element of the GS preorder, while it may be strictly dominated according to the $I_{L}$ index, as illustrated by the example below.

Regarding the weighted sum index, we only consider the most natural weights, i.e. either male marginal, female marginal or the average of the two. In all cases, one can easily check that any change in $(m, n, r)$ that increases AM in the GS sense also increase the weighted likelihood.

\subsection{A simple example}

We remain in the $2 \times 2$ case and still ignore singles. Assume we compare two tables as in Table 5, corresponding for instance to two different cohorts in the same economy.

The matching tables are represented in Table 5. In economic terms, between cohorts $\mathrm{A}$ and $\mathrm{B}$, the number of educated people has drastically increased (from $10 \%$ to $50 \%$ ). In cohort A, matching is positive assortative in the usual sense (more people on the diagonal than would obtain under random matching); yet, $70 \%$ of college graduate men (women) marry a less educated spouse. Cohort B, on the contrary, displays perfect assortative matching: all college men marry college women, and conversely. 
Table 5: Changes in Assortative Matching - An Example

A: Table $(.1, .1, .03)$

\begin{tabular}{|c|c|c|}
\hline$w \backslash^{h}$ & $\mathrm{C}$ & $\mathrm{HS}$ \\
\hline $\mathrm{C}$ & .03 & .07 \\
\hline $\mathrm{HS}$ & .07 & .83 \\
\hline
\end{tabular}

$B$ : Table $(.5, .5, .5)$

\begin{tabular}{|c|c|c|}
\hline$w \backslash^{h}$ & $\mathrm{C}$ & $\mathrm{HS}$ \\
\hline $\mathrm{C}$ & .5 & 0 \\
\hline $\mathrm{HS}$ & 0 & .5 \\
\hline \hline
\end{tabular}

In such a setting, the Perfect Assortative Matching condition would require that the degree of PAM is found to increase (or at least not to decrease) when moving from A to B. We now consider how the various criteria developed above assess the variation.

Standard SEV We start with the basic SEV framework. For cohort A,

$$
I_{S E V}(.1, .1, .03)=1.625
$$

which is positive, indicating PAM; regarding cohort B, the index is infinite. Therefore B displays more AM than A in the $S E V$ sense - as expected from the fact that the $S E V$ criterion satisfies the Perfect Assortative Matching condition.

Generalized Separable The relevant ratios are for cohort A are

$$
\frac{r}{m}=\frac{r}{n}=.3, \quad \frac{1-n-m+r}{1-n}=\frac{1-n-m+r}{1-m}=.92
$$


while for cohort B we obtain

$$
\frac{r}{m}=\frac{r}{n}=\frac{1-n-m+r}{1-n}=\frac{1-n-m+r}{1-m}=1
$$

The four inequalities are satisfied, and we conclude that B displays more AM than A in the GS sense.

The $\chi^{2}$ criterion Regarding the $\chi^{2}$ criterion, cohort A gives

$$
\chi^{2}(A)=4.94 \times 10^{-2}
$$

while for cohort B

$$
\chi^{2}(B)=1
$$

and the degree of PAM increases from A to B - reflecting again the fact that the $\chi^{2}$ criterion satisfies the Perfect Assortative Matching condition.

Minimum distance and perfect-random normalization A similar conclusion obtains with the minimum distance index (which coincides here with the perfect-random normalization criterion). For Table A, we have

$$
I_{M D}(.1, .1, .03)=0.22
$$

while for cohort B

$$
I_{M D}(.5, .5, .5)=1
$$

Again, the degree of PAM increases from A to B, due to the fact that the minimum distance criterion satisfies the Perfect Assortative Matching condition.

The likelihood criterion The weighted likelihood criterion gives similar results. Due to symmetry, the three usual weightings give the same weighted index of 1.22 for Table A; all indices are equal to 2 for Table B. 
However, the unweighted criterion gives an opposite answer. Indeed, the indices are

$$
I_{L}(.1, .1, .03)=3 \text { and } I_{L}(.5, .5, .5)=2
$$

According to that criterion, therefore, cohort B displays less assortative matching than A.

Discussion The previous example illustrates a clear dichotomy between the various criteria under consideration. All measures but one conclude that AM has increased from $\mathrm{A}$ to $\mathrm{B}$, while the unweighted Likelihood criterion concludes the opposite.

How can this discrepancy be explained? A major challenge faced by any comparison of assortativeness between matching tables is to disentangle the mechanical effect of changes in the marginals (i.e., in our case, the distribution of men and women across education classes) from genuine shifts in economic motivations for educational assortative mating. The SEV criterion and its various generalizations achieve this by referring to an explicit model; in particular, changes in 'preferences for assortativeness' directly translate into changes in the supermodularity of the structural matrix $Z$, irrespective of the distribution of education by genders. Neither the $\chi^{2}$ nor the minimum distance nor the weighted likelihood criteria rely on a similar, structural interpretation. Yet, their basic, qualitative properties are similar to those of the separable approaches; in particular, they satisfy the two properties defined above. From that perspective, the status of the unweighted Likelihood approach appears to be somewhat problematic. That a matching table characterized by perfect assortative matching can be found to display strictly less AM than one that does not clearly raises a serious issue in terms of its ability to measure changes in AM.

\section{Beyond the $2 \times 2$ case}

The previous approaches can be extended to take singles into account, and generalized to any ('small') number of categories, although this generalization raises specific issues.

\subsection{The $k \times k$ case}

Consider first the case of more educational categories. As mentioned earlier, assortativeness is a local property; matching patterns can be assortative at the top of the distribution but not at the 
bottom (or conversely). In particular, a comparison of assortativeness between two $k \times k$ Tables can be performed in various ways, potentially leading to different conclusions.

The issue can readily be illustrated in a $3 \times 3$ context; as it turns out, all the difficulties generated in the general case are already present in this setting. Keeping the same interpretation as before, assume three possible levels of education: college graduates and above $(\mathrm{C})$, high school graduates (HS) and high school drop-outs (D). The matching patterns conditional on marriage are given in Table 6 , the Table is now: Here, $m_{1}$ (resp. $n_{1}$ ) denotes the fraction of college educated women Table 6: The $\mathrm{k} \times \mathrm{k}$ case

\begin{tabular}{l|ccc}
\hline \hline$w \backslash h$ & $\mathrm{C}$ & $\mathrm{HS}$ & $\mathrm{D}$ \\
\hline $\mathrm{C}$ & $r_{1}$ & $a$ & $n_{1}-r_{1}-a$ \\
$\mathrm{HS}$ & $b$ & $r_{2}$ & $n_{2}-r_{2}-b$ \\
$\mathrm{D}$ & $m_{1}-r_{1}-b$ & $m_{2}-r_{2}-a$ & $1+r_{1}+r_{2}+a+b-\left(m_{1}+m_{2}+n_{1}+n_{2}\right)$ \\
\hline \hline
\end{tabular}

(men), and $m_{2}\left(n_{2}\right)$ of high-school graduates women (men). There are $r_{1}$ (resp. $\left.r_{2}\right)$ couples where both spouses are college (HS) graduates, and total population size is again normalized to 1.

\subsubsection{Subtables}

When it comes to comparing assortativeness, several perspectives can be adopted. One may concentrate on the more educated categories (C and HS) and ask whether, among couples whose spouses both belong to this set, we see more assortative matching. This amounts to considering the top left diagonal subtable: and applying any of the previously defined criteria. For the SEV framework, for

Table 7 : The $2 \times 2$ case

\begin{tabular}{c|cc}
$w \backslash^{h}$ & $\mathrm{C}$ & $\mathrm{HS}$ \\
\hline \hline $\mathrm{C}$ & $r_{1}$ & $a$ \\
$\mathrm{HS}$ & $b$ & $r_{2}$ \\
\hline \hline
\end{tabular}

instance, one would compute the (sub)index:

$$
I^{C, H S}=\ln \left(\frac{r_{1} r_{2}}{a b}\right)
$$


Of course, one could also consider any alternative, diagonal subtable, and define the subindices:

$$
\begin{aligned}
I^{H S, D} & =\ln \left(\frac{r_{2}\left(1+r_{1}+r_{2}+a+b-\left(m_{1}+m_{2}+n_{1}+n_{2}\right)\right)}{\left(m_{2}-r_{2}-a\right)\left(n_{2}-r_{2}-b\right)}\right) \\
\text { and } I^{C, D} & =\ln \left(\frac{r_{1}\left(1+r_{1}+r_{2}+a+b-\left(m_{1}+m_{2}+n_{1}+n_{2}\right)\right)}{\left(m_{1}-r_{1}-b\right)\left(n_{1}-r_{1}-a\right)}\right)
\end{aligned}
$$

The value of these indices can change independently of each other. When comparing two Tables $A$ and $B$, for instance, we may find that $I^{C, H S}$ is larger for $A$ (suggesting that $A$ displays more PAM than $B$, in the SEV sense, at the top of the distribution) while $I^{H S, D}$ is larger for $B$ (suggesting the opposite conclusion at the bottom).

\subsubsection{Merged tables}

Alternatively, one could exclusively concentrate on one particular education category - say, college and above - and 'merge' the remaining categories into a single, 'everybody else' class. This would lead to the following, $2 \times 2$ merged table: Using for instance the standard SEV approach, the

Table 8: The College versus Others

\begin{tabular}{c|cc}
\hline \hline$w \backslash^{h}$ & $\mathrm{C}$ & Others \\
\hline $\mathrm{C}$ & $r_{1}$ & $n_{1}-r_{1}$ \\
Others & $m_{1}-r_{1}$ & $1+r_{1}-m_{1}-n_{1}$ \\
\hline \hline
\end{tabular}

('C-specific') index would then be:

$$
I^{C}=\ln \left(\frac{r_{1}\left(1+r_{1}-m_{1}-n_{1}\right)}{\left(m_{1}-r_{1}\right)\left(n_{1}-r_{1}\right)}\right)
$$

Here, an increase in $I^{C}$ could be interpreted as 'college educated people are more likely to marry their own' - as opposed to marrying anybody else.

Of course, the previous constructs are by no means specific to the SEV criterion. Whatever criterion is applied, one can consider the 3 (or, in the $k \times k$ case, the $k(k-1) / 2$ ) diagonal submatrices, as well as the 3 (or $k$ ) category-specific merged matrices, and either compute the corresponding indices or, in the GS approach, compare the corresponding ratios. Yet, the SEV context, unlike the 
others, offers a precise, structural interpretation of these constructs, that is described in the next subsection.

\subsubsection{Relationships}

A natural question, at this point, is whether there exists a relationship between these various indices. In particular, assume that, when comparing two $3 \times 3$ tables $A$ and $B$, we find that the three subindices $I^{C, H S}, I^{H S, D}$ and $I^{C, D}$ are larger in Table $A$. Does it imply that $A$ displays more PAM than $B$ ? In particular, should we expect college educated people to be more likely to marry their own in Table $A$ - that is, the $C$-specific index $I^{C}$ to be larger for $A$ ?

Even in the standard SEV case and the $3 \times 3$ context, the answer to that question happens to be negative. A counterexample is provided in the next subsection; but it is useful, at this point, to provide a more theoretical intuition for this result in the SEV case.

Note, first, that in the SEV context merged matrices have a structural interpretation. Consider a woman $i$ with college education, who can choose between a husband in $C, H S$ or $D$. As we have seen, this amounts to choosing the maximum between $U^{C C}+\varepsilon_{i}^{C}, U^{C H S}+\varepsilon_{i}^{H S}$ and $U^{C D}+\varepsilon_{i}^{D}$, where the $\varepsilon_{i}^{X}(X=C, H S, D)$ are EV distributed.

If we merge $H S$ and $D$, the choice is now between $U^{C C}+\varepsilon_{i}^{C}$ and $\max \left\{U^{C H S}+\varepsilon_{i}^{H S}, U^{C D}+\varepsilon_{i}^{D}\right\}$. By a standard property of the EV distribution, this max is itself extreme value distributed. That is, the choice is now between $U^{C C}+\varepsilon_{i}^{C}$ and $U^{C O}+\varepsilon_{i}^{O}$, where $\varepsilon_{i}^{O}$ follows an extreme value distribution and where

$$
U^{C O}=\ln \left(\exp \left(U^{C H S}\right)+\exp \left(U^{C D}\right)\right)
$$

It follows that the $2 \times 2$ merged table can be analyzed under the same assumptions as in Section 2 .

Actually, as long as we disregard singles, a similar argument can be directly applied to the $Z$ matrices themselves. To see why, simply note that, following Proposition 3 , the $3 \times 3$ initial table 
can be generated by the structural matrix:

$$
Z=\left(\begin{array}{ccc}
\ln r_{1} & \ln a & \ln \left(n_{1}-r_{1}-a\right) \\
\ln b & \ln r_{2} & \ln \left(n_{2}-r_{2}-b\right) \\
\ln \left(m_{1}-r_{1}-b\right) & \ln \left(m_{2}-r_{2}-a\right) & \ln \left(\begin{array}{c}
1+r_{1}+r_{2}+a+b \\
-\left(m_{1}+m_{2}+n_{1}+n_{2}\right)
\end{array}\right)
\end{array}\right)
$$

while the $2 \times 2$ merged table can be generated by the $2 \times 2$ structural matrix:

$$
\bar{Z}=\left(\begin{array}{cc}
\ln r_{1} & \ln \left(n_{1}-r_{1}\right) \\
\ln \left(m_{1}-r_{1}\right) & \ln \left(1+r_{1}-m_{1}-n_{1}\right)
\end{array}\right)
$$

In particular, one can check that:

$$
\bar{Z}^{C O}=\ln \left(n_{1}-r_{1}\right)=\ln \left(\exp Z^{C H S}+\exp Z^{C D}\right)
$$

and that a similar relationship obtains for the other coefficients.

While an explicit relationship exists between the $Z_{\mathrm{s}}$ and the $\bar{Z}_{\mathrm{s}}$, this relationship is highly non linear; and there is no reason to expect that supermodularity of some of the submatrices in $Z$ would imply supermodularity of $\bar{Z}$. Indeed, the following subsection provides a counterexample to this claim.

\subsubsection{A counterexample}

Consider the $3 \times 3$ Tables

\begin{tabular}{l|ccc}
\hline \hline$w \backslash^{h}$ & $C$ & $H S$ & $D$ \\
\hline$C$ & .2 & .1 & .1 \\
$H S$ & .1 & .21 & .09 \\
$D$ & .1 & .09 & .01 \\
\hline \hline
\end{tabular}

Table $A$

\begin{tabular}{c|ccc}
\hline \hline${ }_{w} \backslash^{h}$ & $C$ & $H S$ & $D$ \\
\hline and $C$ & .18 & .11 & .1 \\
$H S$ & .11 & .29 & .01 \\
$D$ & .1 & .01 & .09 \\
\hline \multicolumn{4}{c}{ Table $B$}
\end{tabular}

Table $B$ 
For Table $A$, we get

$$
I^{C, H S}=1.435, I^{C, D}=-1.609, I^{H S, D}=-1.35 \text { and } I^{C}=0.693
$$

while for Table $B$ :

$$
I^{C, H S}=1.462, I^{C, D}=.482, I^{H S, D}=5.565 \text { and } I^{C}=0.490
$$

All diagonal subindices are larger for $B$ than for $A$; yet, the $C$-specific index $I^{C}$ is smaller.

\subsection{Introducing singles}

Secondly, one can take into account singles as well as married couples. In the standard, SEV model, the conclusion is clear: taking singles into account does not change the value of any of indices just defined. The argument is straightforward. The structural matrix $\left(Z^{I J}\right)$ is estimated from global matching patterns, including singlehood. The estimation process thus provides values of the structural parameters $Z^{I J}$ that depend on the whole marital pattern, including the number of singles in each category; these estimates would vary should the number of singles change, even when the (conditional) table of matched couples remains unchanged. The specific properties of extreme value distributions however imply that the estimate of the supermodular cores - i.e., the second cross differences $Z^{I J}+Z^{K L}-Z^{I L}-Z^{K J}$ for all $1 \leq I<K \leq k$ and all $1 \leq J<L \leq k$ - only depend on the distribution of matched couples, not on the distribution of singles. Since the SEV indices are directly related to these supermodular cores, they are not affected either. In practice, the formal expression of the indices defined above only depend on the number of married couples in each of the four cells of the matching table; the index conditional on marriage is the same as the unconditional index.

This property, however, is specific to the extreme value case. For general distributions, changes in the number of singles affect the estimated supermodular core, therefore the corresponding assortativeness indices. In other words, unconditional matching patterns do not necessarily coincide with matching patterns conditional on marriage. When it comes to comparing assortativeness between different tables, two claims like 'educated people are more likely to marry their own' and 
'educated people who marry are more likely to marry their own' should be distinguished - unless one is adopting the SEV criterion.

In summary, when comparing two Tables $A$ and $B$, consider the following three claims:

1. College educated people are more likely to marry their own in $A$ than in $B$

2. College educated people who marry are more likely to marry their own in $A$ than in $B$

3. College educated people who marry a (College or High School) educated partner are more likely to marry their own in $A$ than in $B$

For the SEV criterion, claims 1 and 2 are equivalent, but they neither imply nor are implied by claim 3. For other criteria, the three claims are independent. They correspond to different perspectives, and therefore may well lead to different conclusions.

\section{The Data}

We bring evidence on changes in assortativeness in marriage by education using the March extract from the US Current Population Survey (CPS) for the years 1962 to 2019. The survey records sociodemographic information for all adults in the household, including date of birth, gender, education, marital status at the time of the interview, as well as unique identifiers for individuals and their spouses, which allows us to link married couples. We study the changing sorting patterns across 10 -year birth cohorts born in the 30s through to the 70s.

One first practical issue is how to define marriage. CPS reports whether households see themselves as married; we use this information to identify married couples. We then discard observations for married couples where the spouses are not living together, which happens in fewer than $2.5 \%$ of all married couples, and where the identifier for spouse is missing, another $0.5 \%$ of all couples. Other family types, including divorced individuals, widows and those never married are classified as singles. The implication is that cohabiting spouses not reporting themselves as married are also classified as singles. From 1995 onwards these can be identified from information on the relationship between any two members of the family; we find that these unions would increase the number of married couples by about $8.5 \%$. 
Ideally we would like to see the long-lasting marital circumstances that reflect the most relevant family arrangements for each individual. However, CPS only provides a snapshot of the life of each respondent, making it difficult to assess the stability of family arrangements. One issue we need to address is that marriages take place over a long age range and tend to happen later in life among the more educated. This implies that the sorting patterns observed early in life provide a biased picture of marital sorting. To minimise this bias, we select the sub-sample of individuals observed at or past 35 years of age, by which marital rates have stabilised for all education groups as shown in Figure 1. To keep the age distribution across cohorts comparable while maximising the gap between the earliest and latest cohorts, we also capped the age range from above, at 44. The latest cohort, born in the 70s, is truncated in birth year 1975 so that the youngest in that cohort are 44 years old in the last observation year.

Figure 1: Marital rates by age, education and gender

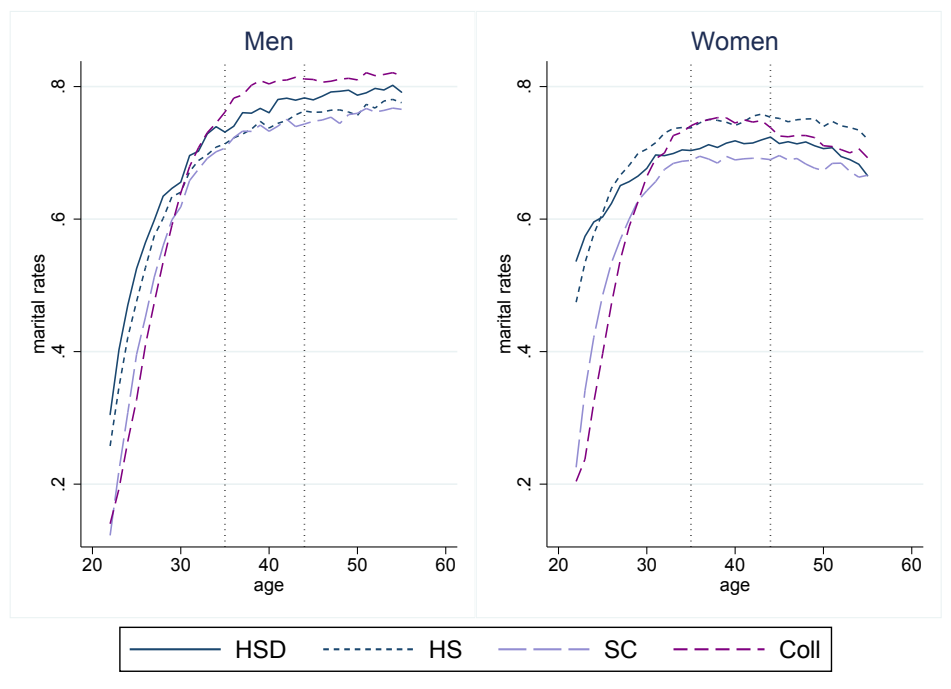

We select the sample of married couples with at least one spouse from the respective cohort and observed while aged 35-44; to that sample we add singles in the same demographic groups. Column 1 of Table 9 shows sample sizes by cohort. Columns 2 and 3 show that the number of single women exceeds the number of single men, consistent with the fact that women are slightly more populous in this age group, especially for the earlier cohorts.

In this paper we study sorting patterns by education split into four groups: High-School Dropouts, which accounts for those who leave education before finishing their 12th grade without 
Table 9: Sample sizes by birth cohort

\begin{tabular}{lrrr}
\hline birth cohort & all families & single men & single women \\
\hline & & & \\
$1930-39$ & 116,899 & 8,555 & 13,326 \\
$1940-49$ & 147,398 & 16,815 & 21,802 \\
$1950-59$ & 173,239 & 28,277 & 33,069 \\
$1960-69$ & 160,474 & 29,983 & 33,345 \\
$1970-75$ & 85,851 & 16,096 & 17,712 \\
& & & \\
\hline
\end{tabular}

any qualifications, High-School graduates, who complete their 12th grade, Some College, representing those who attended college but did not graduate from a 4-year degree, and College graduates, for 4-year or more college graduates. ${ }^{6}$

Figure 2 shows how the distribution of education changed over time, by gender. Some marked changes in education attainment are evident from the figure, with the share of college educated individuals increasing sharply for both men and women. Moreover, the concentration of women in the top education group is lower than that of men at the start of the period, while the reverse is true by the end of it. These changes imply that direct comparisons of sorting patters across cohorts do not reveal the direction of change in assortativeness in marriage by education.

\section{Marital sorting by education}

Marital rates have dropped markedly over time, particularly for those with lower levels of formal education. Figure 3 shows this. Marital rates are slightly lower for women than for men reflecting a relative shortage of men that is more pronounced in the earlier part of the period, for older cohorts. The fall in marital rates has been less pronounced for college graduates, and came to an halt in the early 2000s. As a consequence, by the end of the period marital rates were about $15 \%$ higher among college graduates than for other education groups.

Figure 4 depicts the rate of homogamous marriages over time among those who marry. The

\footnotetext{
${ }^{6}$ In a separate set of results, we also split the latter group further, into 4-year College degrees and Post-graduate degrees. The results are in all similar to those in the paper. Estimates available from the authors upon request.
} 
Figure 2: Distribution of education among those aged 35-44 over time, by gender

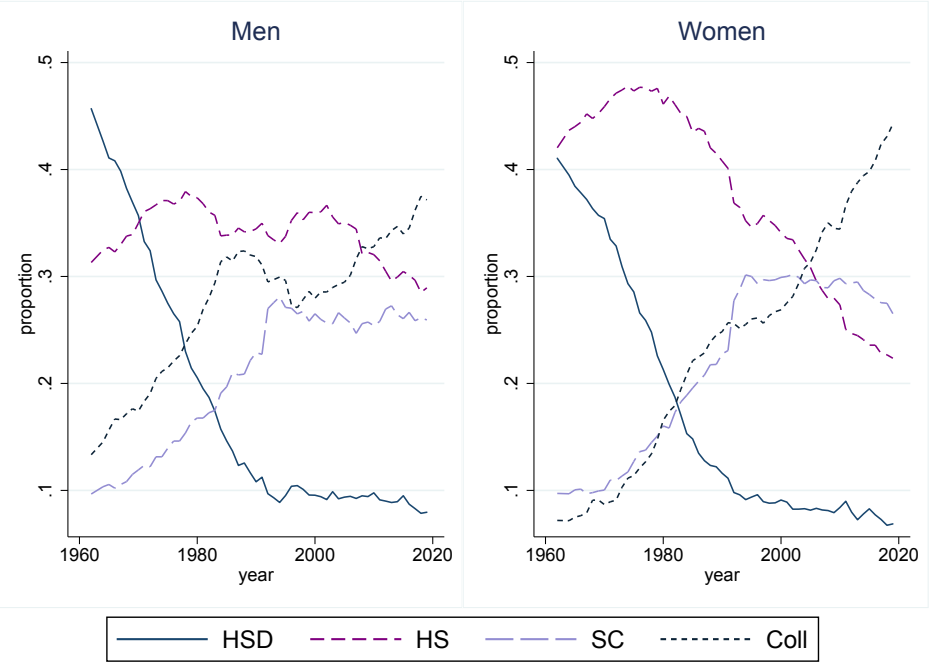

Figure 3: Marital rates among 35-44 years old over time, by education and gender

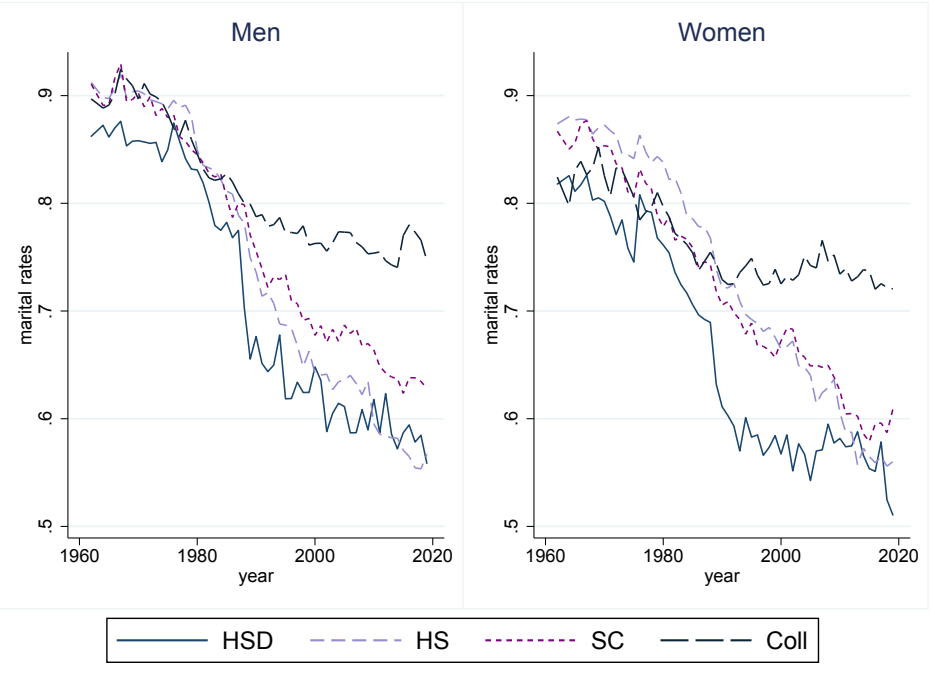

figure shows an initial drop in the proportion of marriages of like with like, which reverses into a sharp increase from the end of the 1980s that continues today (Shen (2019) reports a similar finding). The earliest cohort we consider is 35-44 years old in calendar years 1965-83, precisely when the probability of homogamous marriages is at its lowest. So our focus will be on the period of rapid increase in the probablity of marrying one's own type. Of course, this figure is no proof of increasing assortativeness. In part, it can be driven by the very substantial changes in the distribution of education on both sides of the marriage market. It may also be driven by changes in who gets married, as suggested by the growing divergence in marital rates between college educated 
Figure 4: Rate of homogamous marriages among 35-44 years old over time, conditional on marriage

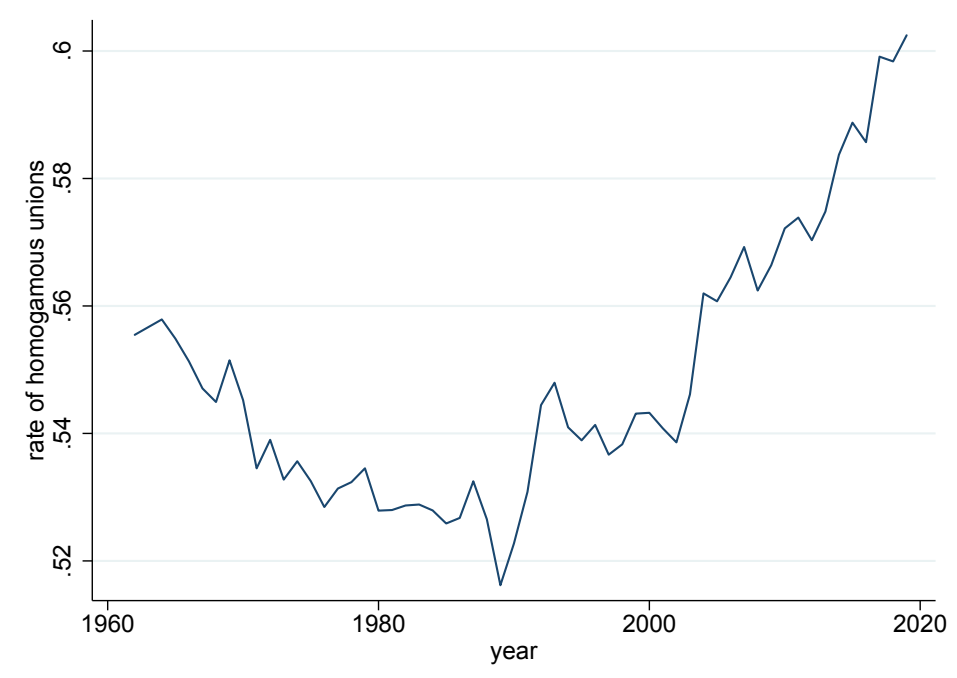

individuals and those with lower levels of education shown in Figure3.

The increasing concentration of marriage among more educated individuals stands out very clearly in the sorting matrices conditional on marriage shown in Table 16. To be concise, the Table focuses on the two cohorts that are furthest apart, born in the 30s and 70s respectively; similar figures for the other cohorts can be found in the Online Appendix. The Table reveals a sharp increase in the proportion of marriages at the high end of the distribution of education, with couples where both spouses are college graduates amounting to $28 \%$ of all couples in the latest cohort. In contrast, the proportion of couples where both spouses have no qualifications declined from $18.4 \%$ to $4.5 \%$ of all unions over the period.

Interestingly, the proportions marrying outside their education class changed very differently for men and women. In particular, it became much less common for college educated men to marry less educated women then themselves, but the same is not true for women. For instance, the probability that a married man with college degree is matched to a wife who is less educated than him dropped from $60 \%$ to $25 \%$ across the two cohorts; for women, however, the probability of a college graduate marrying down actually increased across the two cohorts, from $28 \%$ to $33 \%$. So younger higheducated married women are more likely to be more educated than their spouses in younger than in older cohorts, while the opposite is true for high educated men. ${ }^{7}$ We now investigate the extent

\footnotetext{
${ }^{7}$ In another paper (Chiappori et al., 2020), we estimated sorting patterns for the UK for the 1945-55 and 1965-75 cohorts. Our results for the UK show similar patterns.
} 
Table 10: Sorting patterns by education and birth cohort for individuals observed aged 35-45, CPS data

\begin{tabular}{lrrrr}
\hline & \multicolumn{4}{c}{ wife's education } \\
\cline { 2 - 5 } husband's & High school & High & Some & $4+$ years \\
education & dropouts & school & Coll & college \\
\hline
\end{tabular}

\begin{tabular}{lrrrr} 
& \multicolumn{4}{c}{ Birth cohort 1930-39 } \\
\cline { 2 - 5 } High school dropout & 18.4 & 11.3 & 1.1 & 0.4 \\
High school & 7.5 & 24.1 & 3.3 & 1.3 \\
Some college & 1.4 & 7.0 & 3.1 & 1.4 \\
4+years college & 0.6 & 6.2 & 5.1 & 7.8
\end{tabular}

\begin{tabular}{lrrrr} 
& \multicolumn{4}{c}{ Birth cohort $1970-79$} \\
\cline { 2 - 5 } High school dropout & 4.5 & 2.4 & 1.2 & 0.5 \\
High school & 2.0 & 13.5 & 7.8 & 5.1 \\
Some college & 0.6 & 4.9 & 11.5 & 8.3 \\
4+years college & 0.2 & 2.5 & 6.5 & 28.4
\end{tabular}

to which these patterns reflect changes in assortativeness.

\section{Empirical results on changing assortativeness in marriage}

Existing evidence on the direction of change in assortativeness in marriage over the recent past is inconclusive. Most studies use US data and some find evidence of increase in assortativeness by education and earnings capacity (Chiappori et al., 2017; Greenwood et al., 2014), others find that assortativeness changed differentially across the distribution of education and declined at the top (Eika et al., 2019), and a recent paper finds that assortativeness did not change monotonically over time, having first dropped until the turn of the millennium, and then increased. The comparison across cohorts, however, is confounded by large changes in the distribution of education that we document above. We have argued that changes in the composition of the marriage market limit 
our ability to assess changes in assortativeness without relying on specific assumptions about the structure of the matching model. Here we apply the measures we discussed to bring new evidence on changes in assortativeness in the US marriage market and what drives them.

To keep the results concise while highlighting the most relevant sorting patterns, we will show estimates for changes in assortativeness along the main diagonal only. In practice, this means that we will study the entire subset of $2 \times 2$ sub-matrices that keep same education marriages in the main diagonal. To this, we will add comparisons for the merged sorting tables, conditional and unconditional on marriage as described in Section 6.

To be precise, for a sorting matrix over 4 levels of education (1 to 4 ) as follows

\begin{tabular}{|c|c|c|c|c|}
\hline man $\backslash^{\text {woman }}$ & 1 & 2 & 3 & 4 \\
\hline 1 & $s_{11}$ & $s_{12}$ & $s_{13}$ & $s_{14}$ \\
\hline 2 & $s_{21}$ & $s_{22}$ & $s_{13}$ & $s_{24}$ \\
\hline 3 & $s_{31}$ & $s_{32}$ & $s_{33}$ & $s_{14}$ \\
\hline 4 & $s_{41}$ & $s_{42}$ & $s_{43}$ & $s_{44}$ \\
\hline
\end{tabular}

we will study changes in assortativeness for each of the following $2 \times 2$ submatrices

\begin{tabular}{|l|l|}
\hline$s_{I I}$ & $s_{I J}$ \\
\hline$s_{J I}$ & $s_{J J}$ \\
\hline
\end{tabular}$\quad$ for $(I, J)=(1,2),(2,3),(3,4),(1,3),(2,4),(1,4)$

and for each of the merge matrices

\begin{tabular}{|c|c|}
\hline \multirow[b]{2}{*}{$\sum_{I \neq I} s_{J I}$} & $\sum_{J \neq I} s_{I J}$ \\
\hline & $\sum_{J, K \neq I} s_{J K}$ \\
\hline
\end{tabular}

The latter set of matrices can be constructed both including and excluding singles as part of the outside option for marrying one's like.

For each of these matrices we calculate indices of assortativeness and compare changes across cohorts. As before, we confine attention to the change in sorting patterns between the oldest and youngest cohorts, born in the 30s and 70s respectively. The full set of comparisons can be found in the Online Appendix. 


\subsection{The generalised separable approach}

We first investigate what can be learned from the generalised criteria set out in Proposition 11. This approach has the advantage of being independent of distributional assumptions about unobserved preference components for type of partner, unlike the SEV approach that depends on the extreme value assumption to have a structural interpretation.

Table 11 shows estimated probabilities of homogamous marriages for women and men of different education levels in the earliest and latest birth cohorts, in columns 1 to 4 and 5 to 8, respectively. It includes estimates for all the $2 \times 2$ submatrices along the main diagonal in the top panel, and for the merged matrices describing the probabilities of marrying your like versus marrying anyone else excluding and including the option of remaining singles, in the middle and bottom panels, respectively. An unambiguous, distribution free, increase (decrease) in assortativeness in a $2 \times 2$ matrix corresponds to the case where the probability of marrying a spouse with the same education as oneself increases (decreases) for both men and women in each of the 2 education groups. In practice, these probabilities are defined as ratios and we look at the direction of change in each of them; changes in the same direction of all four ratios identify an unambiguous change in assortativeness.

What is clear from the tables is that unambiguous changes in assortativeness are very uncommon. Indeed, the comparison of the four ratios is inconclusive in all cases. In one single case, shown in the first row of the Table corresponding to the submatrix describing sorting between high-school dropouts and high school graduates, there is strong evidence of an increase in assortativeness: all indices increase except for the one in columns 1 and 5, but the drop there is small and not statistically significant at usual levels. For other groups, the most general set of structural criteria is inconclusive.

To advance, we further impose that the random component of preferences for the education of the spouse follows an extreme value distribution. Under this assumption, Proposition 3 and the discussion that follows it relate changes in the supermodular core of a $2 \times 2$ surplus matrix to

changes in the SEV index: an increase in that index identifies more assortativeness, while a drop identifies less assortativeness. We then compare the results from the SEV approach to those from other indices that have no structural interpretation. 
Table 11: Generalised structural criteria - proportion of homogamous marriages

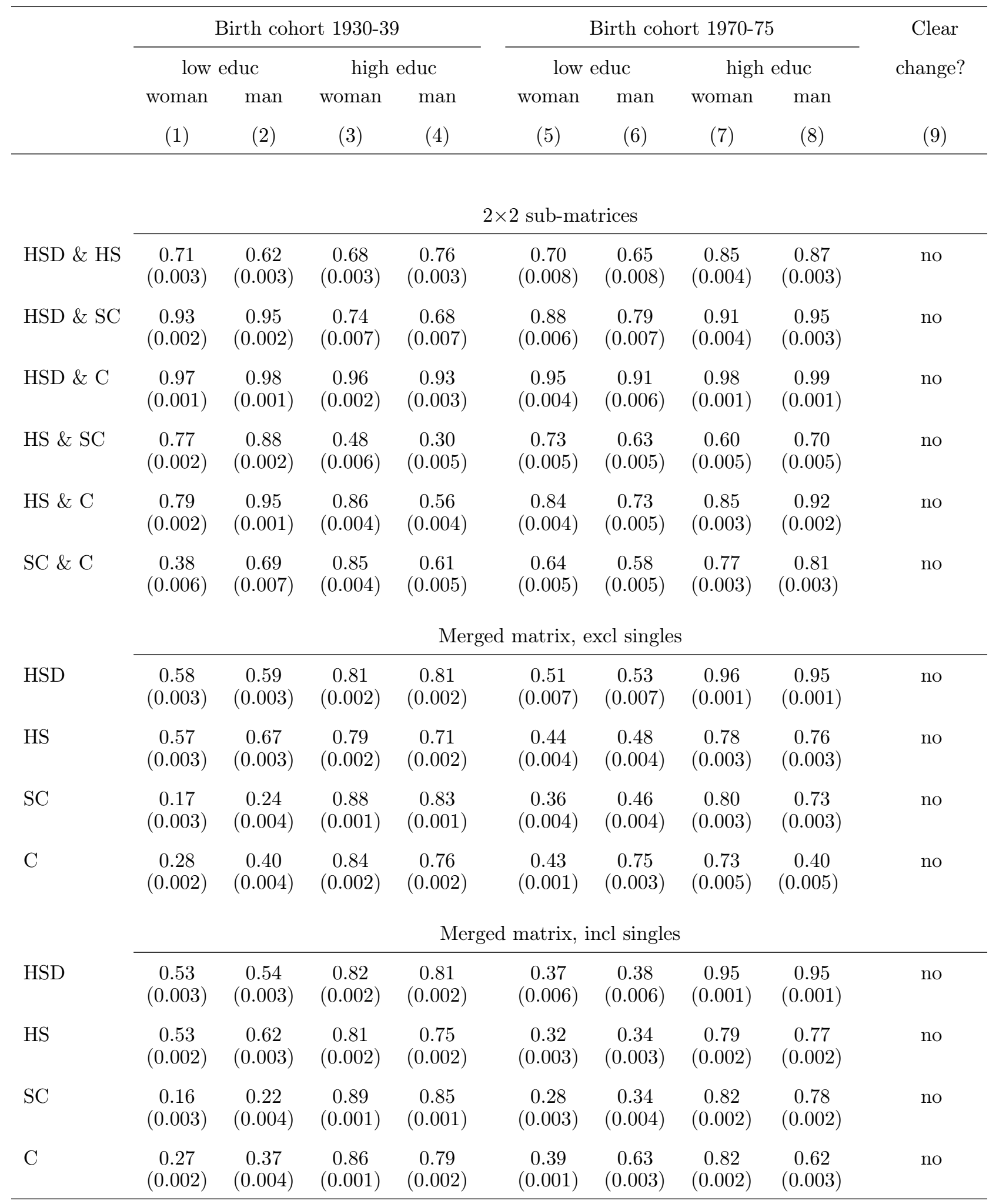

The indices in columns 1 to 4 and 5 to 8 are defined in Proposition 11, calculated for the earliest birth cohort (1930-39) and for the latest (1070-75), respectively. The top panel shows estimates for all $2 \times 2$ submatrices along the main diagonal; the middle panel shows estimates for the matrices comparing marrying ones equal versus marrying anyone else; the bottom panel includes singles in the outside option. 'HSD', 'HS', 'SC' and 'C' are short-hand for high-school dropouts, high school, some college and college graduates, respectively. Bootstraped standard errors in parentheses. 


\subsection{The SEV approach}

Panel A of Table 12 reports the differences in the supermodular core of all submatrices that keep homogamous unions in the main diagonal. The two bottom panels report similar differences for the merged matrices, first excluding singles from the outside option of homogamous marriages at each education level in Panel B, and then including singles in Panel C. The Tables also report the $p$-value for the two-sided significance test, corrected for multiple hypothesis using the stepdown method (Romano and Wolf (2005), Romano et al. (2008), Romano and Wolf (2016)). The test used bootstrapped standard errors based on 1,000 repetitions.

Table 12: Testing changes in marital assortativeness by education between birth cohorts 1930-39 and 1970-75 using the separable extreme value index

\begin{tabular}{lcccccc}
\hline & \multicolumn{7}{c}{ Panel A: $2 \times 2$ submatrices } \\
& HSD \& HS & HS \& SC & SC \& C & HSD \& SC & HS \& C & HSD \& C \\
\cline { 2 - 7 } diff. across cohorts & 0.893 & 0.246 & 0.580 & 0.645 & 0.216 & 0.470 \\
adjusted $p$-value & 0.000 & 0.000 & 0.000 & 0.000 & 0.000 & 0.001 \\
\hline
\end{tabular}

Panel B: merged tables excl. singles

\begin{tabular}{lcccc} 
& HSD & HS & SC & C \\
\cline { 2 - 5 } diff. across cohorts & 1.312 & -0.559 & 0.396 & -0.013 \\
adjusted $p$-value & 0.000 & 0.000 & 0.000 & 0.690
\end{tabular}

Panel B: merged tables incl. singles

\begin{tabular}{lcccc} 
& HSD & HS & SC & C \\
\cline { 2 - 5 } diff. across cohorts & 0.841 & -1.010 & 0.108 & 0.267 \\
adjusted $p$-value & 0.000 & 0.000 & 0.003 & 0.000
\end{tabular}

'HSD', 'HS', 'SC' and 'C' are labels for the 4 education groups, respectively high-school dropouts, high school, some college and college graduates. Panel A describes test results for the comparison of all $2 \times 2$ submatrices keeping homogamous couples in the main diagonal. The columns' labels for the panel identify the two education groups being contrasted. Panels B and C describe test results for the merge tables along the main diagonal, respectively excluding and including singles in the outside option of marrying one's equal. The columns' labels identify the separate category. In each panel, first row shows estimates of the difference in the SEV index between the latest and earliest cohorts. The next row shows the $p$-values for 2 -sided significance testing adjusted for multiple hypothesis considering all 14 tests together. The test uses bootstrapped standard errors based on 1,000 repetitions. 
Tests for the $2 \times 2$ submatrices indicate that assortativeness has increased significantly on all margins being compared. The figures, however, are not as clear once the merged tables are compared, something we show can happen in Section 6.1.3. Whether or not singles are included, these comparisons show a drop in the odds of high-school graduates to marry their like. For all other education groups, however, the odds of homogamous marriages have either increased significantly or not changed significantly over the period.

\subsection{Other measures of assortativeness}

Table 20 shows similar comparisons using each of the other indices of assortativeness discussed before. The first six columns in the Table display estimates for each of the $2 \times 2$ sumbmarices keeping homogamous marriages in the main diagonal. These results are fully aligned with those obtained for the SEV index, reinforcing the view that assortativeness increased in all these margins over the period. 
Table 13: Testing changes in marital assortativeness by education birth cohorts 1930-39 and 1970-75 using other indices

\begin{tabular}{|c|c|c|c|c|c|c|c|c|c|c|c|c|c|c|}
\hline & \multicolumn{6}{|c|}{$2 \times 2$ submatrices } & \multicolumn{8}{|c|}{ merged matrices } \\
\hline & \multirow{2}{*}{$\begin{array}{l}\text { HSD } \\
\& \text { HS }\end{array}$} & \multirow{2}{*}{$\begin{array}{r}\mathrm{HS} \\
\& \mathrm{SC}\end{array}$} & \multirow{2}{*}{$\begin{array}{r}\text { SC } \\
\& C\end{array}$} & \multirow{2}{*}{$\begin{array}{l}\text { HSD } \\
\& \text { SC }\end{array}$} & \multirow{2}{*}{$\begin{array}{r}\mathrm{HS} \\
\& \mathrm{C}\end{array}$} & \multirow{2}{*}{$\begin{array}{l}\text { HSD } \\
\& C\end{array}$} & \multicolumn{4}{|c|}{ conditional on marriage } & \multicolumn{4}{|c|}{ including singles } \\
\hline & & & & & & & HSD & HS & $\mathrm{SC}$ & $\mathrm{C}$ & HSD & HS & $\mathrm{SC}$ & $\mathrm{C}$ \\
\hline & \multicolumn{14}{|c|}{ Panel A: $X^{2}$} \\
\hline diff. across cohorts & 0.135 & 0.064 & 0.097 & 0.162 & 0.112 & -0.009 & 0.066 & -0.084 & 0.026 & 0.005 & -0.015 & -0.110 & 0.008 & 0.033 \\
\hline \multirow[t]{2}{*}{ adjusted $p$-value } & 0.000 & 0.000 & 0.000 & 0.000 & 0.000 & 0.336 & 0.000 & 0.000 & 0.000 & 0.025 & 0.010 & 0.000 & 0.000 & 0.000 \\
\hline & \multicolumn{14}{|c|}{ Panel B: Minimum Distance } \\
\hline diff. across cohorts & 0.123 & 0.097 & 0.022 & 0.139 & -0.036 & 0.004 & 0.079 & -0.178 & 0.131 & 0.106 & -0.022 & -0.280 & 0.049 & 0.165 \\
\hline \multirow[t]{2}{*}{ adjusted $p$-value } & 0.000 & 0.000 & 0.181 & 0.000 & 0.000 & 0.462 & 0.000 & 0.000 & 0.000 & 0.000 & 0.011 & 0.000 & 0.000 & 0.000 \\
\hline & \multicolumn{14}{|c|}{ Panel C: Likelihood ratio } \\
\hline diff. across cohorts & 0.785 & 0.241 & 0.299 & 1.621 & 1.009 & 5.024 & 4.101 & -0.029 & 0.109 & -0.304 & 3.305 & -0.347 & -0.024 & -0.127 \\
\hline \multirow[t]{2}{*}{ adjusted $p$-value } & 0.000 & 0.000 & 0.000 & 0.000 & 0.000 & 0.000 & 0.000 & 0.244 & 0.001 & 0.000 & 0.000 & 0.000 & 0.417 & 0.000 \\
\hline & \multicolumn{14}{|c|}{ Panel D: Weighted sum index } \\
\hline diff. across cohorts & 0.147 & 0.114 & 0.144 & 0.115 & 0.086 & -0.005 & 0.077 & -0.141 & 0.113 & 0.016 & -0.022 & -0.243 & 0.047 & 0.090 \\
\hline adjusted $p$-value & 0.000 & 0.000 & 0.000 & 0.000 & 0.000 & 0.349 & 0.000 & 0.000 & 0.000 & 0.032 & 0.011 & 0.000 & 0.000 & 0.000 \\
\hline
\end{tabular}

'HSD', 'HS', 'SC' and 'C' are labels for the 4 education groups, respectively high-school dropouts, high school, some college and college graduates. Estimates in the first 6 columns are for the comparison of all $2 \times 2$ submatrices keeping homogamous couples in the main diagonal; the next 4 columns show test results for merged tables excluding singles and the last 4 columns show similar test results including singles. In each panel, the first row shows estimates of the difference in the respective index between the latest and earliest cohorts. The second row shows the $p$-values for 2 -sided significance testing adjusted for multiple hypothesis considering all 14 tests together. The test uses bootstrapped standard errors based on 1,000 repetitions. 
The next 8 columns detail results from the comparison of merged tables. Here the figures are consistent with those obtained for the SEV index in most but not all cases. Specifically, there is supporting evidence of a drop in the probability of homogamous marriages among HS graduates and an increase among those with some but not completed college education. The evidence for other education groups is more mixed. Accounting for singles leads to negative changes in most indices for high-school dropouts, although the change is small (but nevertheless statistically significant at conventional levels) in all negative cases. For college graduates, the likelihood ratio test also suggests a drop in the probability of marrying their own, in line with findings reported in Eika et al. (2019). This evidence, however, is not corroborated by any of the other measures.

\section{Counterfactual analysis}

One key advantage of the structural approach, highlighted in Ciscato and Weber (2020) and Chiappori et al. (2020), is that it explicitly links the observed marital patterns with the economic drivers of sorting, which are the values of matching one's like as compared to marrying someone else or not marrying. Under the SEV assumptions, the deterministic surplus from marriage and how it is shared between the two spouses can be determined from the equilibrium sorting patterns. In turn, the surplus matrix can be used to determine the sorting patterns in equilibrium. What this allows us to do is to determine the matching patterns that would result from combining the preferences and incentives implicit in the sorting patterns of one population, call it cohort 1, and the distribution of education of another population, cohort 2. We say that the sorting matrix obtained with such an exercise has the same level of assortativeness as the original sorting matrix for cohort 1 because it is generated by the same preferences. The two matrices, however, will not be identical if the distribution of education is different across the two cohorts.

To demonstrate how we construct the counterfactual sorting matrix for cohort 2 using preferences from cohort 1, suppose there are $N$ education types indexed by $I$ for men and $J$ for women, with 0 representing remaining single. ${ }^{8}$ Denote by $p_{I J}$ the proportion of men who are type $I$ and marry a woman of type $J$ for $I=1, \ldots, N$ and $J=0, \ldots, N$. Similarly, $q_{I J}$ is the proportion of women who are type $J$ and marry a man of type $I$ for $I=0, \ldots, N$ and $J=1, \ldots, N$. Under the SEV

\footnotetext{
${ }^{8}$ For alternative structural approaches to measuring the effects of changes in sorting and empirical results in other contexts see Fernández (2002); Fernández et al. (2005) amongst others.
} 
conditions, it is easy to show that the surplus of a match $(I, J)$ is

$$
Z_{I J}=\ln \left(\frac{p_{I J} q_{I J}}{p_{I 0} q_{0 J}}\right) \quad \text { for } I, J=1, \ldots, N
$$

under the normalization $Z_{I 0}=Z_{0 J}=0$.

Let $\left(T_{m}, T_{w}\right)$ be the number of men and women in the market, respectively. Then $q_{I J}$ equals $p_{I J} T_{m} / T_{w}$ and we can rearrange equation 7 to obtain

$$
\begin{aligned}
p_{I J} & =\exp \left\{\frac{Z_{I J}}{2}\right\} \sqrt{p_{I 0}} \sqrt{q_{0 J}} \sqrt{\frac{T_{w}}{T_{m}}} \\
\text { and } q_{I J} & =\exp \left\{\frac{Z_{I J}}{2}\right\} \sqrt{p_{I 0}} \sqrt{q_{0 J}} \sqrt{\frac{T_{m}}{T_{w}}} .
\end{aligned}
$$

Denote by $P_{I}$ and $Q_{J}$ the measure of men of type $I$ and women of type $J$, respectively. These can be expressed as follows:

$$
\begin{aligned}
P_{I} & =p_{I 0}+\sum_{J=1}^{N} p_{I J} \\
Q_{J} & =q_{0 J}+\sum_{I=1}^{N} q_{I J}
\end{aligned}
$$

Using equations 8 and 9 to replace $\left(p_{I J}, q_{I J}\right)$ in the above yields

$$
\begin{gathered}
p_{I 0}+\sum_{J=1}^{N} \exp \left\{\frac{Z_{I J}}{2}\right\} \sqrt{p_{I 0}} \sqrt{q_{0 J}} \sqrt{\frac{T_{w}}{T_{m}}}-P_{I}=0 \text { for } I=1, \ldots, N \\
q_{0 J}+\sum_{I=1}^{N} \exp \left\{\frac{Z_{I J}}{2}\right\} \sqrt{p_{I 0}} \sqrt{q_{0 J}} \sqrt{\frac{T_{m}}{T_{w}}}-Q_{J}=0 \text { for } J=1, \ldots, N
\end{gathered}
$$

which is a system of $2 N$ equations in $2 N$ unknowns, $\left(p_{I 0}, q_{0 J}, I, J=1, \ldots, N\right)$. Equations 8 to 11 determine the sorting patterns $\left(p_{I J}, q_{I J}\right)$ for all $I$ and $J$ from the structural surplus matrix $Z$, the marginal distributions of education $\left(P_{I}, Q_{J}\right)$ and the ratio of men to women $T_{m} / T_{w}$. One can, therefore, combine the preferences of one population, described by $Z$, with the distributions of education and gender of another population, described by $\left(P_{I}, Q_{J}\right)$ and $T_{m} / T_{w}$, to construct counterfactual sorting matrices.

Using this procedure, we provide further evidence on changes in assortativeness by comparing 
the sorting patterns for the cohort born in the 70s with what it would have been had preferences and incentives for sorting been equal to those shown by the 30s cohort. In this exercise, we keep the distributions of education and gender fixed at the level of the 70s cohort.

Table 14 shows that the value of marriage dropped markedly between the earliest and the latest cohorts. Indeed, while almost $24 \%$ of men born in the 70 s are not married at ages $35-44$ (last row of Table, column 1), only half of those would be singles had preferences for marriage remained at the levels observed for the 30's cohort. Similar changes are observed for women. Low educated women experienced especially pronounced drops in the value of marriage. This is revealed by the difference between the observed and counterfactual singlehood rates for them: had preferences remained intact, the likelihood of them remaining single would be between a fifth and a seventh of what actually happened. The one exception is college educated women, who would be $20 \%$ more likely to be single had the value of marriage not increased for them.

Table 14: Counterfactual simulations - rate of singlehood by education

\begin{tabular}{|c|c|c|c|c|}
\hline & \multicolumn{2}{|c|}{ 1970's cohort } & \multicolumn{2}{|c|}{$\begin{array}{c}\text { Counterfactual } \\
\text { using } \\
\text { 1930's preferences }\end{array}$} \\
\hline & men & women & men & women \\
\hline High school dropout & 27.58 & 31.27 & 12.39 & 6.57 \\
\hline High school & 28.80 & 30.23 & 15.18 & 3.95 \\
\hline Some college & 25.75 & 28.91 & 16.44 & 13.71 \\
\hline $4+$ years college & 16.40 & 18.48 & 6.18 & 22.67 \\
\hline all & 23.62 & 25.39 & 12.16 & 14.17 \\
\hline
\end{tabular}

Table 14 shows how sorting patterns conditional on marriage have changed as a consequence of changes in the value of marrying different classes of spouses. The most significant differences are for marriages with at least one spouse being a college graduate. The contrast between observed and counterfactual patterns shows that homogamous marriages between college graduates would 
have been $5 \%$ less frequent had preferences for marrying different types not changed. The Table also clearly shows that college graduated men are less likely to marry women who are less educated than themselves than they would in the counterfactual scenario, while the opposite is true for college graduated women. This pattern is also evident for other education groups, with the sorting patterns under 1930's preferences showing less concentration of marriages above the main diagonal relative to the observed patterns for the 1970s birth cohort, and more concentration below the main diagonal. This shows how changes in the value of marrying different spouses led to a drop in the frequency of 'classical households', where the husband is more educated than the wife, and an increase in the frequency of 'modern households', where the wife is more educated.

Table 15: Conterfactual simulations - marital sorting conditional on marriage

\begin{tabular}{|c|c|c|c|c|c|c|c|c|}
\hline & \multicolumn{4}{|c|}{ 1970's cohort } & \multicolumn{4}{|c|}{$\begin{array}{c}\text { Counterfactual using } \\
\text { 1930's preferences }\end{array}$} \\
\hline & HSD & HS & $\mathrm{SC}$ & $\mathrm{C}$ & HSD & HS & $\mathrm{SC}$ & $\mathrm{C}$ \\
\hline High school dropout & 4.50 & 2.44 & 1.17 & 0.46 & 4.02 & 3.31 & 1.12 & 0.56 \\
\hline High school & 1.96 & 13.53 & 7.79 & 5.11 & 3.42 & 14.68 & 7.17 & 4.13 \\
\hline Some college & 0.63 & 4.90 & 11.55 & 8.28 & 0.98 & 6.57 & 10.25 & 7.01 \\
\hline $4+$ years college & 0.23 & 2.51 & 6.52 & 28.42 & 0.23 & 3.41 & 9.96 & 23.16 \\
\hline
\end{tabular}

'HSD', 'HS', 'SC' and 'C' are labels for the 4 education groups, respectively high-school dropouts, high school, some college and college graduates.

\section{Concluding Remarks}

There has been an increased interest in understanding how sorting in the marriage market has changed. Specifically, is it the case that the tendency to marry someone with a similar level of education has increased? While this question should be easy to answer it turns out that it is anything but that. The complications arise from the change in the distribution of education among both men and women. For example, the proportion of college graduated women now exceeds that of men. Indeed, various ad hoc indices of changes in sorting have led to opposing conclusions, particularly at the top of the education distribution. 
To address these issues we develop a structural approach to measuring changes in sorting. We note that changes in sorting are directly linked to changes in the benefits of marrying ones own type relative to marrying a different type - i.e. it relates to the structure of the surplus. Based on that observation and building on Choo and Siow (2006), we derive a parametric criterion for changes in sorting that relates directly to a model of the marriage market. We then consider a more general distribution-free index, also linked directly to a structural interpretation. The nonparametric nature of our index only leads to a partial ordering of matching patterns.

Based on our structural approach, and recognizing the fact that simple indices not directly related to a model can be useful and indeed widely used, we derive two minimal criteria that any index should satisfy. We show that most such indices do satisfy these criteria and indeed provide similar conclusions to our structural index when we consider changes in the data. One exception is the Likelihood criterion, which violates one of our conditions, and also leads to opposite conclusions about changes in sorting at the top of the distribution.

Our empirical analysis considers long term changes in marital sorting by education in the US based on CPS data. First, we apply our index and others that have been suggested in the literature to compare changes in between the 1930's cohort and the 1970's. With all the indices that provide a complete ordering, other than the likelihood one, we find that sorting right at the top of the distribution has increased. Our generalized distribution-free index, however gives ambiguous results, meaning that it is not possible to rank the two matching patterns independently of any assumptions on the distribution of the random component of preferences.

We then follow an alternative approach where we keep preferences and incentives to sort the same as they were for the 1930s cohort and we simulate how sorting would have looked like under the current distribution of education. Again we find that under the 1930s preferences and incentives we should obtain less sorting at the top.

Understanding how sorting changes is critically important for many key economic and social issues, including inequality and the intergenerational transmission of human capital. However, it is important to view sorting through the lens of a model to avoid important pitfalls in its measurement. 


\section{References}

Abbott, B., G. Gallipoli, C. Meghir, and G. L. Violante (2019). Education policy and intergenerational transfers in equilibrium. Journal of Political Economy 127(6), 2569-2624.

Becker, G. S. (1981). A Treatise on the Family. Harvard University Press.

Berry, S., J. Levinsohn, and A. Pakes (1995). Automobile prices in market equilibrium. Econometrica $63(4), 841-890$.

Chiappori, P. A. (2017). Matching with transfers: The economics of love and marriage. Princeton University Press.

Chiappori, P. A., M. C. Dias, S. Crossman, and C. Meghir (2020). Changes in assortative matching and inequality in income: Evidence for the uk. Fiscal Studies.

Chiappori, P.-A., M. C. Dias, and C. Meghir (2018). The marriage market, labor supply, and education choice. Journal of Political Economy 126(S1), S26-S72.

Chiappori, P.-A., M. Iyigun, and Y. Weiss (2009, December). Investment in schooling and the marriage market. American Economic Review 99(5), 1689-1713.

Chiappori, P. A., B. Salanié, and Y. Weiss (2017). Partner choice, investment in children, and the marital college premium. American Economic Review 10\%, 175-201.

Choo, E. and A. Siow (2006). Who marries whom and why. Journal of Political Economy 114, $175-201$.

Ciscato, E. and S. Weber (2020). The role of evolving marital preferences in growing income inequality. Journal of Population Economics 33, 307-347.

Dupuy, A. and A. Galichon (2014). Personality traits and the marriage market. Journal of Political Economy 122(6), 1271-1319.

Eika, L., M. Mogstad, and B. Zafar (2019). Educational assortative mating and household income inequality. Journal of Political Economy $12 \%$.

Fernández, R. (2002). Education, segregation and marital sorting: theory and an application to the uk. European Economic Review 46(6), 993 - 1022. ISOM.

Fernández, R., N. Guner, and J. Knowles (2005). Love and Money: A Theoretical and Empirical Analysis of Household Sorting and Inequality*. The Quarterly Journal of Economics 120(1), 273-344.

Fernández, R. and R. Rogerson (2001). Sorting and long-run inequality. The Quarterly Journal of Economics 116(4), 1305-1341.

Galichon, A. and B. Salanié (2019). Cupid's invisible hand: Social surplus and identification in matching models. mimeo, Columbia University.

Graham, B. (2011). Econometric methods for the analysis of assignment problems in the presence of complementarity and social spillovers. Handbook of Social Economics 1B: 965 - 1052 (J. Benhabib, M. O. Jacksons and Alberto Bisin, Eds.) Amsterdam: North-Holland. 
Graham, B. (2013). Comparative static and computational methods for an empirical one-to-one transferable utility matching model. Advances in Econometrics 31, 151-179.

Greenwood, J., N. Guner, and J. Knowles (2003). More on marriage, fertility, and the distribution of income. International Economic Review 44, 827-862.

Greenwood, J., N. Guner, G. Kocharkov, and C. Santos (2014). Marry your like: Assortative mating and income inequality. American Economic Review: Papers and Proceedings 104, 348-353.

Liu, H. and J. Lu (2006). Measuring the degree of assortative mating. Economics Letters 92, $317-322$.

Mare, R. (1991). Five decades of educational assortative mating. American Sociological Review 56, $15-32$.

McFadden, D. (1981). Econometric models of probabilistic choice. In C. Manski and D. McFadden (Eds.), Structural Analysis of Discrete Data and Econometric Applications, pp. 198-272. Cambridge: MIT Press.

Romano, J., A. Shaikh, and M. Wolf (2008). Formalized data snooping based on generalized error rates. Econometric Theory 24, 404-447.

Romano, J. and M. Wolf (2005). Stepwise multiple testing as formalized data snooping. Econometrica 73, 1237?1282.

Romano, J. and M. Wolf (2016). Efficient computation of adjusted p-values for resampling-based stepdown multiple testing. Statistics and Probability Letters 113, 38-40.

Schwartz, C. and R. Mare (2005). Five decades of educational assortative mating. Demography 42, 621-646.

Shen, J. (2019). (non-)marital assortative mating and the closing of the gender gap in education. mimeo, Princeton University. 


\section{Online Appendix}

\section{Proof of Proposition 3}

Proposition 1 implies that for any woman $i \in I$, the probability $P_{I J}$ of being matched with a man in category $J$ is given by:

$$
\begin{aligned}
P_{I J} & =\operatorname{Pr}\left(U^{I J}+\varepsilon_{i}^{J} \geq U^{I K}+\varepsilon_{i}^{K}\right) & \forall K \\
& =\operatorname{Pr}\left(\varepsilon_{i}^{J}-\varepsilon_{i}^{K} \geq U^{I K}-U^{I J}\right) & \forall K
\end{aligned}
$$

Symmetrically, that for any man $j \in J$, the probability $Q_{I J}$ of being matched with a woman in category $I$ is given by:

$$
\begin{aligned}
Q_{I J} & =\operatorname{Pr}\left(V^{I J}+\nu_{j}^{I} \geq V^{K J}+\nu_{j}^{K}\right) & \forall K \\
& =\operatorname{Pr}\left(\nu_{j}^{I}-\nu_{j}^{K} \geq V^{K J}-V^{I J}\right) & \forall K
\end{aligned}
$$

Using the logistic assumption:

$$
P_{I J}=\frac{\exp U^{I J}}{\sum_{K} \exp U^{I K}} \quad \text { and } \quad Q_{I J}=\frac{\exp V^{I J}}{\sum_{K} \exp V^{K J}}
$$

where the $U^{I J}$ (resp. $V^{I J}$ ) are defined up to $2 N$ normalizations; here, $N=2$, which gives 4 normalizations. For Table $(m, n, r)$, these relationships give

$$
\begin{aligned}
& P_{11}=\frac{r}{m}=\frac{\exp U^{11}}{\exp U^{11}+\exp U^{12}}, P_{12}=1-P_{11} \\
& P_{21}=\frac{n-r}{1-m}=\frac{\exp U^{21}}{\exp U^{21}+\exp U^{22}}, P_{22}=1-P_{21}
\end{aligned}
$$

which, after the normalization $U^{11}=U^{21}=0$, can be inverted as:

$$
\begin{aligned}
U^{12} & =\ln \left(\frac{m-r}{r}\right), \\
U^{22} & =\ln \left(\frac{1+r-m-n}{n-r}\right)
\end{aligned}
$$

Similarly,

$$
\begin{aligned}
Q_{11} & =\frac{r}{n}=\frac{\exp V^{11}}{\exp V^{11}+\exp V^{21}}, Q_{21}=1-Q_{11} \\
Q_{12} & =\frac{m-r}{1-n}=\frac{\exp V^{21}}{\exp V^{12}+\exp V^{22}}, Q_{22}=1-Q_{12}
\end{aligned}
$$

which, after the same normalization $V^{11}=V^{12}=0$, can be inverted as:

$$
\begin{aligned}
V^{21} & =\ln \left(\frac{n-r}{r}\right) \\
V^{22} & =\ln \left(\frac{1+r-m-n}{m-r}\right)
\end{aligned}
$$


In the end:

$$
\begin{aligned}
& Z^{11}=0, Z^{22}=\ln \left(\frac{(1+r-m-n)^{2}}{(n-r)(m-r)}\right) \\
& Z^{12}=\ln \left(\frac{m-r}{r}\right) \text { and } Z^{21}=\ln \left(\frac{n-r}{r}\right)
\end{aligned}
$$

Finally, one can readily see that any alternative normalization than the one used here $\left(U^{11}=U^{21}=\right.$ $V^{11}=V^{12}=0$ ) would give different values for the $Z^{i j}$, but would not change the cross difference $Z^{11}+Z^{22}-Z^{12}-Z^{21}$. For instance, the form

$$
\begin{aligned}
Z^{11} & =2 \ln r, Z^{12}=2 \ln (m-r), Z^{21}=2 \ln (n-r) \\
\text { and } Z^{22} & =2 \ln (1+r-m-n)
\end{aligned}
$$

generates the same matching patterns.

\section{Proof of Proposition 8}

Define $F$ and $G$ as the cdf of $\left(\varepsilon_{i}^{1}-\varepsilon_{i}^{2}\right)$ and $\left(\nu_{j}^{1}-\nu_{j}^{2}\right)$. With the same notations as above:

$$
\begin{aligned}
& P_{11}=1-F\left(U^{12}-U^{11}\right) \Rightarrow U^{12}-U^{11}=F^{-1}\left(1-P_{11}\right) \\
& P_{21}=1-F\left(U^{22}-U^{21}\right) \Rightarrow U^{22}-U^{21}=F^{-1}\left(1-P_{21}\right)
\end{aligned}
$$

and under the normalization $U^{11}=U^{21}=0$

$$
\begin{aligned}
& P_{11}=1-F\left(U^{12}\right) \Rightarrow U^{12}=H\left(1-P_{11}\right) \\
& P_{21}=1-F\left(U^{22}\right) \Rightarrow U^{22}=H\left(1-P_{21}\right)
\end{aligned}
$$

where $H=F^{-1}$ is an increasing function mapping $R$ to $[0,1]$.

Similarly, under the normalization $V^{11}=V^{12}=0$

$$
\begin{aligned}
& Q_{11}=1-G\left(V^{21}\right) \Rightarrow V^{21}=K\left(1-Q_{11}\right) \\
& Q_{12}=1-G\left(V^{22}\right) \Rightarrow V^{22}=K\left(1-Q_{12}\right)
\end{aligned}
$$

where $K=G^{-1}$ is also increasing from $R$ to $[0,1]$.

Let $\Delta$ denote the supermodular core, which for given $m$ and $n$ depends on $r$; we thus have that:

$$
\begin{aligned}
\Delta(r) & =U^{22}+V^{22}-U^{12}-V^{21} \\
& =H\left(1-P_{21}\right)+K\left(1-Q_{12}\right)-H\left(1-P_{11}\right)-K\left(1-Q_{11}\right)
\end{aligned}
$$

Here,

$$
P_{11}=\frac{r}{m}, P_{21}=\frac{n-r}{1-m}, Q_{11}=\frac{r}{n} \text { and } Q_{12}=\frac{m-r}{1-n}
$$

so that

$$
\Delta(r)=H\left(\frac{1+r-m-n}{1-m}\right)+K\left(\frac{1+r-m-n}{1-n}\right)-H\left(\frac{m-r}{m}\right)-K\left(\frac{n-r}{n}\right)
$$


For given $m$ and $n$, this expression is increasing in $r$; therefore $\Delta(r) \geq \Delta(\bar{r})$ if and only if $r \geq \bar{r}$, which closes the proof.

\section{Proof of Proposition 11}

We want to show that shifting from $(m, n, r)$ to $(\bar{m}, \bar{n}, \bar{r})$ increases $\Gamma$, where, as above:

$$
\begin{aligned}
\Gamma(m, n, r) & =H\left(\frac{1+r-m-n}{1-m}\right)-H\left(\frac{m-r}{m}\right)+K\left(\frac{1+r-m-n}{1-n}\right)-K\left(\frac{n-r}{n}\right) \\
& =D(m, n, r)+E(m, n, r)
\end{aligned}
$$

where

$$
\begin{aligned}
& D(m, n, r)=H\left(1-\frac{n-r}{1-m}\right)-H\left(1-\frac{r}{m}\right) \text { and } \\
& E(m, n, r)=K\left(\frac{1+r-m-n}{1-n}\right)-K\left(\frac{n-r}{n}\right)
\end{aligned}
$$

Since $H$ is increasing, the inequalities in Proposition 11 imply that

$$
H\left(1-\frac{n-r}{1-m}\right) \geq H\left(1-\frac{\bar{n}-\bar{r}}{1-\bar{m}}\right) \geq H\left(1-\frac{\bar{r}}{\bar{m}}\right) \geq H\left(1-\frac{r}{m}\right)
$$

where the second inequality comes from Positive Assortative Matching. Therefore

$$
D(m, n, r) \geq D(\bar{m}, \bar{n}, \bar{r})
$$

The same holds for $E$.

Conversely, assume that one of the previous inequalities is violated - say,

$$
H\left(1-\frac{n-r}{1-m}\right)<H\left(1-\frac{\bar{n}-\bar{r}}{1-\bar{m}}\right)
$$

Then one can find some small enough $\delta$ such that

$$
H\left(1-\frac{n-r}{1-m}\right)<H\left(1-\frac{\bar{n}-\bar{r}}{1-\bar{m}}\right)-3 \delta
$$

and one can choose two increasing functions $H$ and $K$ such that

$$
\begin{aligned}
H\left(1-\frac{\bar{r}}{\bar{m}}\right) & \geq H\left(1-\frac{r}{m}\right)-\delta \\
K\left(1-\frac{\bar{m}-\bar{r}}{1-\bar{n}}\right) & \geq K\left(1-\frac{m-r}{1-n}\right)-\delta \text { and } \\
K\left(1-\frac{\bar{r}}{\bar{n}}\right) & \geq K\left(1-\frac{r}{n}\right)-\delta
\end{aligned}
$$

It follows that

$$
D(m, n, r)<D(\bar{m}, \bar{n}, \bar{r})-2 \delta \text { and } E(m, n, r) \leq E(\bar{m}, \bar{n}, \bar{r})+2 \delta
$$


therefore

$$
\Gamma(m, n, r)<\Gamma(\bar{m}, \bar{n}, \bar{r})
$$

\section{Proof of Proposition 15}

Define:

$$
x=\frac{r}{m}, y=\frac{r}{n}, z=\frac{1-n-m+r}{1-m}
$$

then

$$
m=\frac{y(1-z)}{x+y-x y-y z}, n=\frac{x(1-z)}{x+y-x y-y z}, r=\frac{x y(1-z)}{x+y-x y-y z}
$$

and let $R$ be the last ratio:

$$
R=\frac{1-n-m+r}{1-n}=\frac{x z(1-y)}{y-x y+x z-y z}
$$

We want Positive Assortative Matching, implying :

$$
r \geq m n \Rightarrow z \geq 1-x
$$

We therefore define the set $S$ as

$$
S=\left\{(x, y, z) \in R^{3} \text { such that } x \geq 0, y \geq 0, z \geq 0 \text { and } x+z \geq 1\right\}
$$

Note in particular that any change $(d x, d y, d z)$ belonging to $S$ increases the first three ratios of Proposition 11.

With these notations,

$$
\chi^{2}=\frac{y(1-y)(x+z-1)^{2}}{y-x y+x z-y z}
$$

and we consider an infinitesimal increase $(d x, d y, d z) \in S$ such that $R$ does not decrease, i.e. such that:

$$
(d x, d y, d z)^{\prime} \cdot R=y z(1-y)(1-z) d x-x z(1-x)(1-z) d y+x y(1-x)(1-y) d z \geq 0
$$

We need to check that for any infinitesimal increase $(d x, d y, d z)$ in $S$ that does not decrease $R, \chi^{2}$ increases as well. Here:

$$
\begin{aligned}
& \frac{\partial\left(\chi^{2}\right)}{\partial x}=\frac{y(1-y)(x+z-1)}{(y-x y+x z-y z)^{2}}\left(y+z-x y+x z-y z-z^{2}\right) \geq 0 \\
& \frac{\partial\left(\chi^{2}\right)}{\partial y}=\frac{(x+z-1)^{2}}{(y-x y+x z-y z)^{2}}\left(x y^{2}+y^{2} z+x z-y^{2}-2 x y z\right) \\
& \frac{\partial\left(\chi^{2}\right)}{\partial z}=\frac{y(1-y)(x+z-1)}{(y-x y+x z-y z)^{2}}\left(x+y-x y+x z-y z-x^{2}\right) \geq 0
\end{aligned}
$$

Note that $\partial \chi^{2} / \partial x$ and $\partial \chi^{2} / \partial z$ are both nonnegative on $S$, whereas $\partial \chi^{2} / \partial y$ may be positive or negative. 
Define the vectors:

$$
\begin{aligned}
& V^{\prime}=(R)^{\prime}=\left(\begin{array}{c}
y z(1-y)(1-z) \\
-x z(1-x)(1-z) \\
x y(1-x)(1-y)
\end{array}\right) \\
& W^{\prime}=\left(\chi^{2}\right)^{\prime}=\left(\begin{array}{c}
y z(1-y)\left(y+z-x y+x z-y z-z^{2}\right) \\
(x+z-1)\left(x y^{2}+y^{2} z+x z-y^{2}-2 x y z\right) \\
y(1-y)\left(x+y-x y+x z-y z-x^{2}\right)
\end{array}\right)
\end{aligned}
$$

Figure 5: Vectors $V$ and $W$

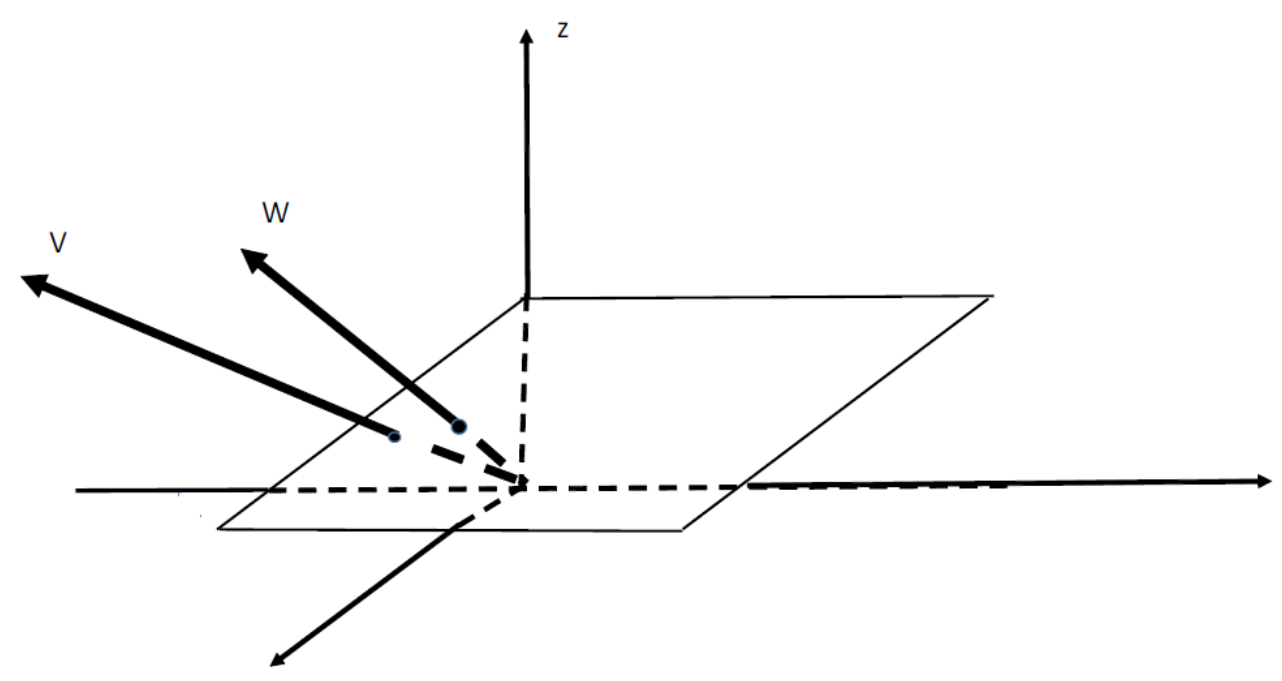

We want that for any vector $v$ with positive components,

$$
v^{\prime} V \geq 0 \Rightarrow v^{\prime} W \geq 0
$$

this is equivalent to showing that $W$ belongs to the convex cone generated by $(1,0,0),(0,1,0),(0,0,1)$ and $V$. If we intersect the cone with the horizontal plan $Z=1$ (Figure 5), which is equivalent to considering the vectors

$$
\begin{aligned}
\bar{V} & =\left(\frac{z(1-z)}{x(1-x)},-\frac{z(1-z)}{y(1-y)}, 1\right) \text { and } \\
\bar{W} & =\left(\frac{\left(y+z-x y+x z-y z-z^{2}\right)}{\left(x+y-x y+x z-y z-x^{2}\right)}, \frac{(x+z-1)\left(x y^{2}+y^{2} z+x z-y^{2}-2 x y z\right)}{y(1-y)\left(x+y-x y+x z-y z-x^{2}\right)}, 1\right)
\end{aligned}
$$

then we want $\bar{W}$ to belong to the convex cone generated by the first unit vector and vector $\bar{V}$ (Figure 6).

This is equivalent to

$$
\frac{(x+z-1)\left(x y^{2}+y^{2} z+x z-y^{2}-2 x y z\right)}{y(1-y)\left(y+z-x y+x z-y z-z^{2}\right)}>-\frac{x(1-x)}{y(1-y)}
$$


Figure 6: Vectors $\bar{V}$ and $\bar{W}$

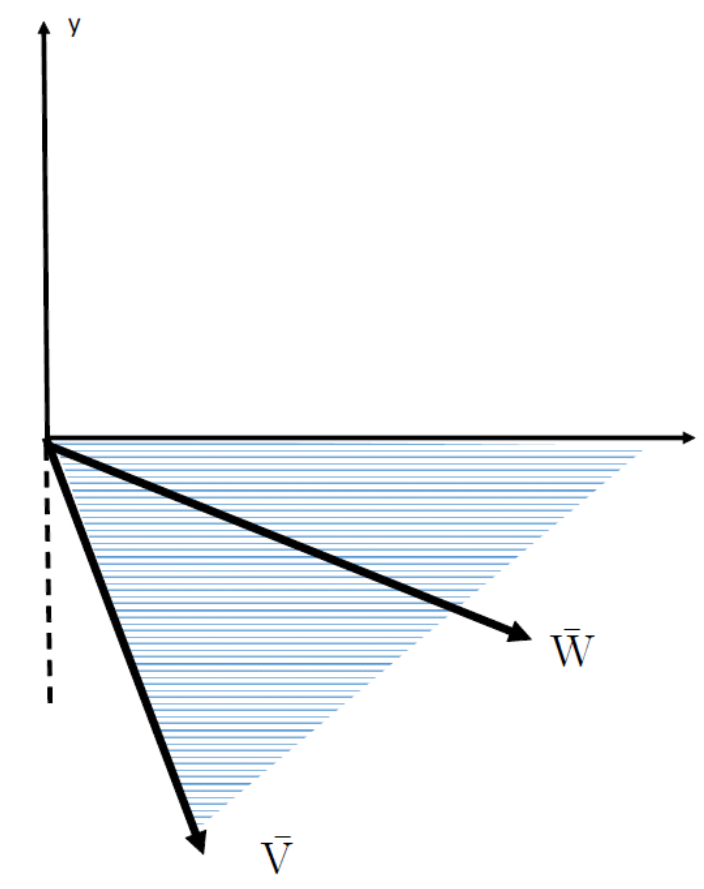

therefore

$$
\frac{(x+z-1)\left(x y^{2}+y^{2} z+x z-y^{2}-2 x y z\right)}{\left(y+z-x y+x z-y z-z^{2}\right)}+x(1-x) \geq 0
$$

It can readily be checked that the left hand side expression is non negative over $S$.

Conversely, one can easily find changes $(d x, d y, d z)$ that increase the $\chi^{2}$ but do not increase all ratios in Proposition 11.

\section{Proof of Proposition 16}

Here,

$$
I_{M D}(m, n, r)=\frac{r-m n}{n-m n}
$$

Again, define:

$$
x=\frac{r}{m}, y=\frac{r}{n}, z=\frac{1-n-m+r}{1-m}
$$

then

$$
I_{M D}=\frac{1}{x} y(x+z-1)
$$

which is increasing in $x, y$ and $z$ on $S$. It follows that a change in $(m, n, r)$ that increases AM in the GS sense, therefore increases $x, y$ and $z$, must increase the minimum distance index. The converse is obviously false; for instance, if one decreases $x$ and increases $z$ by the same $\varepsilon, I_{M D}$ increases, but the new Table does not display more AM than the initial one in the GS sense since $x$ is smaller. 


\section{Sorting Tables for 10-year birth cohorts from the 1930s to the 1970s}

Table 16: Sorting patterns by education and birth cohort for individuals observed aged 35-45, CPS data

\begin{tabular}{|c|c|c|c|c|}
\hline \multirow[b]{2}{*}{$\begin{array}{l}\text { husband's } \\
\text { education }\end{array}$} & \multicolumn{4}{|c|}{ wife's education } \\
\hline & $\begin{array}{r}\text { High school } \\
\text { dropouts }\end{array}$ & $\begin{array}{r}\text { High } \\
\text { school }\end{array}$ & $\begin{array}{r}\text { Some } \\
\text { Coll }\end{array}$ & $\begin{array}{r}+ \text { years } \\
\text { college }\end{array}$ \\
\hline & \multicolumn{4}{|c|}{ Birth cohort 1930-39 } \\
\hline High school dropout & 18.4 & 11.3 & 1.1 & 0.4 \\
\hline High school & 7.5 & 24.1 & 3.3 & 1.3 \\
\hline Some college & 1.4 & 7.0 & 3.1 & 1.4 \\
\hline \multirow{2}{*}{$4+$ years college } & 0.6 & 6.2 & 5.1 & 7.8 \\
\hline & \multicolumn{4}{|c|}{ Birth cohort 1940-49 } \\
\hline High school dropout & 8.7 & 7.2 & 0.9 & 0.32 \\
\hline High school & 5.0 & 23.5 & 4.7 & 2.11 \\
\hline Some college & 1.1 & 8.5 & 5.8 & 2.76 \\
\hline \multirow[t]{2}{*}{$4+$ years college } & 0.4 & 6.3 & 7.5 & 14.76 \\
\hline & \multicolumn{4}{|c|}{ Birth cohort 1950-59 } \\
\hline High school dropout & 4.7 & 3.8 & 1.0 & 0.26 \\
\hline High school & 3.0 & 19.9 & 7.1 & 2.96 \\
\hline Some college & 0.9 & 9.0 & 10.8 & 4.84 \\
\hline \multirow{2}{*}{$4+$ years college } & 0.2 & 4.9 & 8.1 & 17.93 \\
\hline & \multicolumn{4}{|c|}{ Birth cohort 1960-69 } \\
\hline High school dropout & 4.3 & 3.0 & 1.1 & 0.36 \\
\hline High school & 2.5 & 7.4 & 8.7 & 4.26 \\
\hline Some college & 0.7 & 6.7 & 12.0 & 6.23 \\
\hline \multirow[t]{2}{*}{$4+$ years college } & 0.2 & 3.4 & 7.1 & 21.60 \\
\hline & \multicolumn{4}{|c|}{ Birth cohort 1970-75 } \\
\hline High school dropout & 4.5 & 2.4 & 1.2 & 0.5 \\
\hline High school & 2.0 & 13.5 & 7.8 & 5.1 \\
\hline Some college & 0.6 & 4.9 & 11.5 & 8.3 \\
\hline $4+$ years college & 0.2 & 2.5 & 6.5 & 28.4 \\
\hline
\end{tabular}




\section{Changes in assortativeness across birth cohorts, from the 1930s to the 1970s}

Table 17: Testing changes in marital assortativeness by education birth cohorts 1930-39 and 1940-49 using various indices

\begin{tabular}{|c|c|c|c|c|c|c|c|c|c|c|c|c|c|c|}
\hline & \multicolumn{6}{|c|}{$2 \times 2$ submatrices } & \multicolumn{8}{|c|}{$\begin{array}{ll}\text { merged matrices } \\
\end{array}$} \\
\hline & \multirow{2}{*}{$\begin{array}{l}\text { HSD } \\
\& \text { HS }\end{array}$} & \multirow{2}{*}{$\begin{array}{r}\mathrm{HS} \\
\& \mathrm{SC}\end{array}$} & \multirow{2}{*}{$\begin{array}{r}\text { SC } \\
\& \mathrm{C}\end{array}$} & \multirow{2}{*}{$\begin{array}{l}\text { HSD } \\
\& \text { SC }\end{array}$} & \multirow{2}{*}{$\begin{array}{r}\mathrm{HS} \\
\& \mathrm{C}\end{array}$} & \multirow{2}{*}{$\begin{array}{r}\text { HSD } \\
\& \mathrm{C}\end{array}$} & \multicolumn{4}{|c|}{ conditional on marriage } & \multicolumn{4}{|c|}{ including singles } \\
\hline & & & & & & & HSD & HS & $\mathrm{SC}$ & $\mathrm{C}$ & HSD & $\mathrm{HS}$ & $\mathrm{SC}$ & $\mathrm{C}$ \\
\hline & \multicolumn{14}{|c|}{ Panel A: SEV } \\
\hline diff. across cohorts & 0.080 & 0.049 & 0.189 & 0.204 & 0.075 & 0.337 & 0.332 & 0.014 & -0.042 & -0.308 & 0.270 & -0.107 & -0.072 & -0.291 \\
\hline adjusted $p$-value & 0.013 & 0.358 & 0.000 & 0.006 & 0.199 & 0.002 & 0.000 & 0.590 & 0.339 & 0.000 & 0.000 & 0.000 & 0.083 & 0.000 \\
\hline \multirow{3}{*}{$\begin{array}{l}\text { diff. across cohorts } \\
\text { adjusted } p \text {-value }\end{array}$} & \multicolumn{14}{|c|}{ Panel B: $X^{2}$} \\
\hline & -0.001 & 0.020 & 0.028 & 0.124 & 0.076 & 0.030 & -0.002 & 0.001 & 0.001 & -0.010 & -0.015 & -0.019 & 0.000 & -0.009 \\
\hline & 0.834 & 0.000 & 0.000 & 0.000 & 0.000 & 0.000 & 0.936 & 0.928 & 0.452 & 0.000 & 0.000 & 0.000 & 0.935 & 0.000 \\
\hline \multirow{3}{*}{$\begin{array}{l}\text { diff. across cohorts } \\
\text { adjusted } p \text {-value }\end{array}$} & \multicolumn{14}{|c|}{ Panel C: Minimum Distance } \\
\hline & -0.006 & 0.029 & 0.019 & 0.070 & -0.013 & 0.008 & -0.002 & 0.003 & 0.011 & -0.044 & -0.022 & -0.033 & 0.004 & -0.036 \\
\hline & 0.772 & 0.016 & 0.590 & 0.000 & 0.289 & 0.332 & 0.738 & 0.819 & 0.309 & 0.000 & 0.000 & 0.000 & 0.866 & 0.000 \\
\hline \multirow{3}{*}{$\begin{array}{l}\text { diff. across cohorts } \\
\text { adjusted } p \text {-value }\end{array}$} & \multicolumn{14}{|c|}{ Panel D: Likelihood ratio } \\
\hline & 0.304 & 0.046 & 0.101 & 0.375 & 0.201 & 1.153 & 0.992 & 0.021 & -0.085 & -0.285 & 0.957 & 0.008 & -0.111 & -0.302 \\
\hline & 0.000 & 0.000 & 0.000 & 0.000 & 0.000 & 0.000 & 0.000 & 0.087 & 0.002 & 0.000 & 0.000 & 0.554 & 0.000 & 0.000 \\
\hline & \multicolumn{14}{|c|}{ Panel E: Weighted sum index } \\
\hline diff. across cohorts & -0.001 & 0.040 & 0.048 & 0.089 & 0.058 & 0.016 & -0.002 & 0.002 & 0.008 & -0.045 & -0.022 & -0.028 & 0.003 & -0.037 \\
\hline adjusted $p$-value & 0.870 & 0.000 & 0.000 & 0.000 & 0.000 & 0.000 & 0.938 & 0.924 & 0.461 & 0.000 & 0.000 & 0.000 & 0.942 & 0.000 \\
\hline
\end{tabular}

Notes: 'HSD', 'HS', 'SC' and 'C' are labels for the 4 education groups, respectively high-school dropouts, high school, some college and college graduates. Estimates in the first 6 columns are for the comparison of all $2 \times 2$ submatrices keeping homogamous couples in the main diagonal; the next 4 columns show test results for merged tables excluding singles and the last 4 columns show similar test results including singles. In each panel, the first row shows estimates of the difference in the respective index between the latest and earliest cohorts. The second row shows the $p$-values for 2 -sided significance testing adjusted for multiple hypothesis considering all 14 tests together. The test uses bootstrapped standard errors based on 1,000 repetitions. 
Table 18: Testing changes in marital assortativeness by education birth cohorts 1940-49 and 1950-59 using various indices

\begin{tabular}{|c|c|c|c|c|c|c|c|c|c|c|c|c|c|c|}
\hline & \multicolumn{6}{|c|}{$2 \times 2$ submatrices } & \multicolumn{8}{|c|}{ merged matrices } \\
\hline & \multirow{2}{*}{$\begin{array}{r}\text { HSD } \\
\& \text { HS } \\
\end{array}$} & \multirow{2}{*}{$\begin{array}{r}\mathrm{HS} \\
\& \mathrm{SC} \\
\end{array}$} & \multirow{2}{*}{$\begin{array}{r}\mathrm{SC} \\
\& \mathrm{C} \\
\end{array}$} & \multirow{2}{*}{$\begin{array}{l}\text { HSD } \\
\& \mathrm{SC} \\
\end{array}$} & \multirow{2}{*}{$\begin{array}{r}\mathrm{HS} \\
\& \mathrm{C} \\
\end{array}$} & \multirow{2}{*}{$\begin{array}{r}\text { HSD } \\
\& \mathrm{C} \\
\end{array}$} & \multicolumn{4}{|c|}{ conditional on marriage } & \multicolumn{4}{|c|}{ including singles } \\
\hline & & & & & & & HSD & HS & $\mathrm{SC}$ & $\mathrm{C}$ & HSD & $\mathrm{HS}$ & $\mathrm{SC}$ & $\mathrm{C}$ \\
\hline & \multicolumn{14}{|c|}{ Panel A: SEV } \\
\hline diff. across cohorts & 0.347 & -0.005 & 0.178 & 0.146 & -0.064 & 0.278 & 0.523 & -0.210 & 0.125 & 0.072 & 0.259 & -0.449 & 0.071 & 0.205 \\
\hline adjusted $p$-value & 0.000 & 0.845 & 0.000 & 0.063 & 0.118 & 0.040 & 0.000 & 0.000 & 0.000 & 0.002 & 0.000 & 0.000 & 0.015 & 0.000 \\
\hline \multirow{3}{*}{$\begin{array}{l}\text { diff. across cohorts } \\
\text { adjusted } p \text {-value }\end{array}$} & \multicolumn{14}{|c|}{ Panel B: $X^{2}$} \\
\hline & 0.038 & 0.015 & 0.041 & 0.008 & 0.022 & -0.002 & 0.015 & -0.034 & 0.007 & 0.004 & -0.020 & -0.057 & 0.004 & 0.011 \\
\hline & 0.000 & 0.000 & 0.000 & 0.683 & 0.000 & 0.737 & 0.011 & 0.000 & 0.000 & 0.000 & 0.000 & 0.000 & 0.000 & 0.000 \\
\hline \multirow{3}{*}{$\begin{array}{l}\text { diff. across cohorts } \\
\text { adjusted } p \text {-value }\end{array}$} & \multicolumn{14}{|c|}{ Panel C: Minimum Distance } \\
\hline & 0.030 & -0.010 & 0.002 & 0.008 & -0.056 & -0.008 & 0.018 & -0.066 & 0.046 & 0.034 & -0.032 & -0.125 & 0.026 & 0.060 \\
\hline & 0.000 & 0.413 & 0.861 & 0.613 & 0.000 & 0.275 & 0.032 & 0.000 & 0.000 & 0.000 & 0.000 & 0.000 & 0.000 & 0.000 \\
\hline \multirow{3}{*}{$\begin{array}{l}\text { diff. across cohorts } \\
\text { adjusted } p \text {-value }\end{array}$} & \multicolumn{14}{|c|}{ Panel D: Likelihood ratio } \\
\hline & 0.467 & 0.087 & -0.048 & 1.024 & 0.164 & 1.863 & 1.837 & -0.009 & 0.015 & 0.003 & 1.315 & -0.152 & 0.007 & 0.106 \\
\hline & 0.000 & 0.000 & 0.000 & 0.000 & 0.000 & 0.000 & 0.000 & 0.775 & 0.778 & 0.911 & 0.000 & 0.000 & 0.736 & 0.000 \\
\hline & \multicolumn{14}{|c|}{ Panel E: Weighted sum index } \\
\hline diff. across cohorts & 0.046 & 0.026 & 0.059 & 0.005 & 0.017 & -0.001 & 0.018 & -0.049 & 0.039 & 0.018 & -0.031 & -0.106 & 0.025 & 0.044 \\
\hline adjusted $p$-value & 0.000 & 0.000 & 0.000 & 0.681 & 0.000 & 0.737 & 0.011 & 0.000 & 0.000 & 0.000 & 0.000 & 0.000 & 0.000 & 0.000 \\
\hline
\end{tabular}

Notes: 'HSD', 'HS', 'SC' and 'C' are labels for the 4 education groups, respectively high-school dropouts, high school, some college and college graduates. Estimates in the first 6 columns are for the comparison of all $2 \times 2$ submatrices keeping homogamous couples in the main diagonal; the next 4 columns show test results for merged tables excluding singles and the last 4 columns show similar test results including singles. In each panel, the first row shows estimates of the difference in the respective index between the latest and earliest cohorts. The second row shows the $p$-values for 2 -sided significance testing adjusted for multiple hypothesis considering all 14 tests together. The test uses bootstrapped standard errors based on 1,000 repetitions. 
Table 19: Testing changes in marital assortativeness by education birth cohorts 1950-59 and 1960-69 using various indices

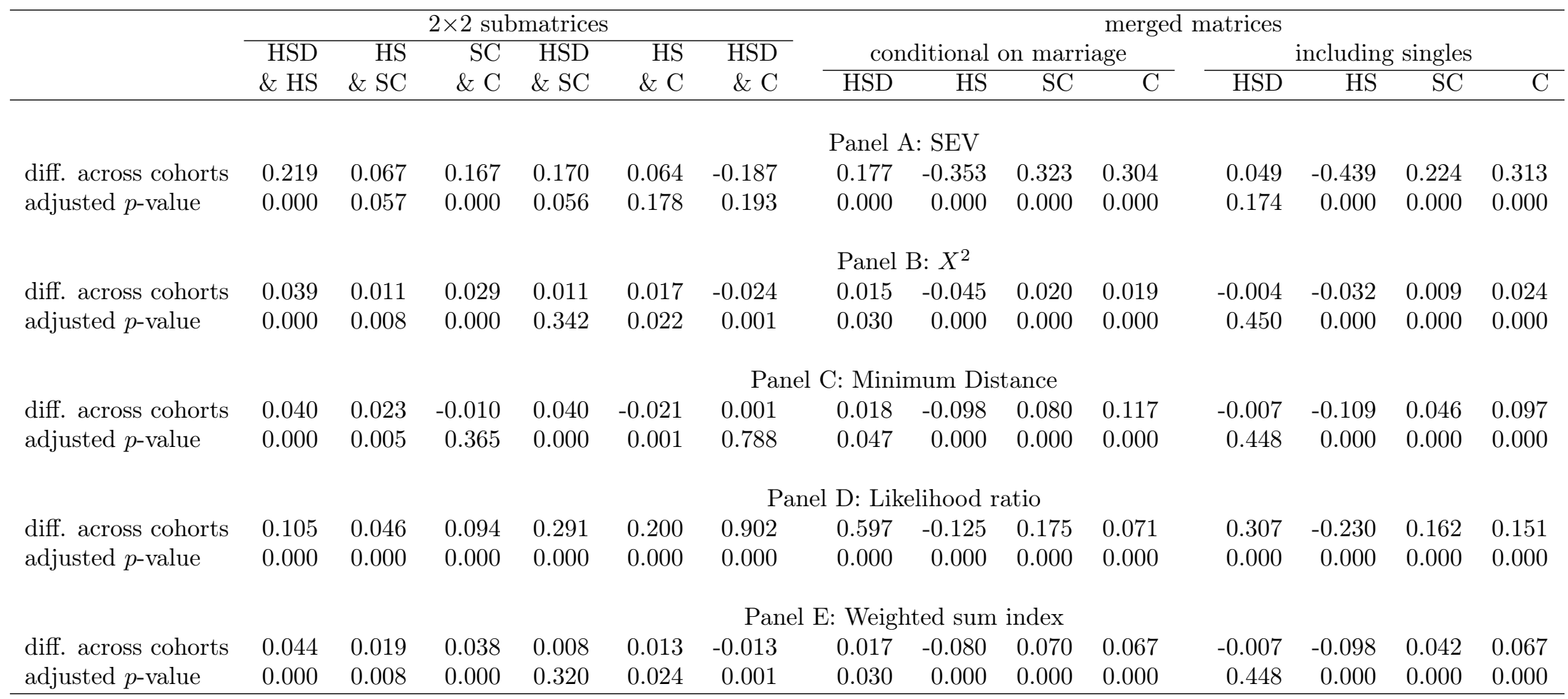

Notes: 'HSD', 'HS', 'SC' and 'C' are labels for the 4 education groups, respectively high-school dropouts, high school, some college and college graduates. Estimates in the first 6 columns are for the comparison of all $2 \times 2$ submatrices keeping homogamous couples in the main diagonal; the next 4 columns show test results for merged tables excluding singles and the last 4 columns show similar test results including singles. In each panel, the first row shows estimates of the difference in the respective index between the latest and earliest cohorts. The second row shows the $p$-values for 2 -sided significance testing adjusted for multiple hypothesis considering all 14 tests together. The test uses bootstrapped standard errors based on 1,000 repetitions. 
Table 20: Testing changes in marital assortativeness by education birth cohorts 1960-69 and 1970-75 using various indices

\begin{tabular}{|c|c|c|c|c|c|c|c|c|c|c|c|c|c|c|}
\hline & \multicolumn{6}{|c|}{$2 \times 2$ submatrices } & \multicolumn{8}{|c|}{ merged matrices } \\
\hline & \multirow{2}{*}{$\begin{array}{r}\text { HSD } \\
\& \mathrm{HS} \\
\end{array}$} & \multirow{2}{*}{$\begin{array}{r}\mathrm{HS} \\
\& \mathrm{SC}\end{array}$} & \multirow{2}{*}{$\begin{array}{r}\mathrm{SC} \\
\& \mathrm{C} \\
\end{array}$} & \multirow{2}{*}{$\begin{array}{l}\text { HSD } \\
\& \text { SC }\end{array}$} & \multirow{2}{*}{$\begin{array}{r}\mathrm{HS} \\
\& \mathrm{C}\end{array}$} & \multirow{2}{*}{$\begin{array}{r}\mathrm{HSD} \\
\& \mathrm{C} \\
\end{array}$} & \multicolumn{4}{|c|}{ conditional on marriage } & \multicolumn{4}{|c|}{ including singles } \\
\hline & & & & & & & HSD & HS & $\mathrm{SC}$ & $\mathrm{C}$ & HSD & HS & $\mathrm{SC}$ & $\mathrm{C}$ \\
\hline \multirow{3}{*}{$\begin{array}{l}\text { diff. across cohorts } \\
\text { adjusted } p \text {-value }\end{array}$} & \multicolumn{14}{|c|}{ Panel A: SEV } \\
\hline & 0.247 & 0.135 & 0.047 & 0.126 & 0.141 & 0.042 & 0.280 & -0.010 & -0.010 & -0.080 & 0.263 & -0.014 & -0.116 & 0.040 \\
\hline & 0.000 & 0.001 & 0.457 & 0.511 & 0.003 & 0.985 & 0.000 & 0.760 & 0.941 & 0.075 & 0.000 & 0.976 & 0.000 & 0.329 \\
\hline \multirow{3}{*}{$\begin{array}{l}\text { diff. across cohorts } \\
\text { adjusted } p \text {-value }\end{array}$} & \multicolumn{14}{|c|}{ Panel B: $X^{2}$} \\
\hline & 0.059 & 0.019 & -0.001 & 0.019 & -0.003 & -0.013 & 0.039 & -0.007 & -0.001 & -0.008 & 0.024 & -0.002 & -0.005 & 0.007 \\
\hline & 0.000 & 0.001 & 0.869 & 0.459 & 0.866 & 0.491 & 0.000 & 0.242 & 0.915 & 0.001 & 0.000 & 0.231 & 0.000 & 0.000 \\
\hline \multirow{3}{*}{$\begin{array}{l}\text { diff. across cohorts } \\
\text { adjusted } p \text {-value }\end{array}$} & \multicolumn{14}{|c|}{ Panel C: Minimum Distance } \\
\hline & 0.060 & 0.056 & 0.012 & 0.022 & 0.054 & 0.003 & 0.044 & -0.019 & -0.007 & -0.001 & 0.039 & -0.012 & -0.027 & 0.044 \\
\hline & 0.000 & 0.000 & 0.372 & 0.141 & 0.000 & 0.841 & 0.000 & 0.085 & 0.788 & 0.956 & 0.000 & 0.150 & 0.000 & 0.000 \\
\hline \multirow{4}{*}{$\begin{array}{l}\text { diff. across cohorts } \\
\text { adjusted } p \text {-value }\end{array}$} & \multicolumn{14}{|c|}{ Panel D: Likelihood ratio } \\
\hline & -0.090 & 0.062 & 0.152 & -0.069 & 0.443 & 1.106 & 0.675 & 0.085 & 0.005 & -0.094 & 0.725 & 0.028 & -0.082 & -0.083 \\
\hline & 0.030 & 0.000 & 0.000 & 0.239 & 0.000 & 0.000 & 0.000 & 0.000 & 0.801 & 0.000 & 0.000 & 0.264 & 0.000 & 0.000 \\
\hline & \multicolumn{14}{|c|}{ Panel E: Weighted sum index } \\
\hline diff. across cohorts & 0.058 & 0.029 & -0.001 & 0.013 & -0.002 & -0.007 & 0.043 & -0.014 & -0.004 & -0.023 & 0.038 & -0.011 & -0.022 & 0.016 \\
\hline adjusted $p$-value & 0.000 & 0.001 & 0.880 & 0.451 & 0.918 & 0.502 & 0.000 & 0.256 & 0.913 & 0.002 & 0.000 & 0.242 & 0.000 & 0.000 \\
\hline
\end{tabular}

Notes: 'HSD', 'HS', 'SC' and 'C' are labels for the 4 education groups, respectively high-school dropouts, high school, some college and college graduates. Estimates in the first 6 columns are for the comparison of all $2 \times 2$ submatrices keeping homogamous couples in the main diagonal; the next 4 columns show test results for merged tables excluding singles and the last 4 columns show similar test results including singles. In each panel, the first row shows estimates of the difference in the respective index between the latest and earliest cohorts. The second row shows the $p$-values for 2 -sided significance testing adjusted for multiple hypothesis considering all 14 tests together. The test uses bootstrapped standard errors based on 1,000 repetitions. 